\title{
FRONT TRACKING FOR SCALAR BALANCE EQUATIONS
}

\author{
K. H. KARLSEN, N. H. RISEBRO, AND J. D. TOWERS
}

\begin{abstract}
We propose and prove convergence of a front tracking method for scalar conservation laws with source term. The method is based on writing the single conservation law as a $2 \times 2$ quasilinear system without a source term, and employ the solution of the Riemann problem for this system in the front tracking procedure. In this way the source term is processed in the Riemann solver, and one avoids using operator splitting. Since we want to treat the resonant regime, classical arguments for bounding the total variation of numerical solutions do not apply here. Instead compactness of a sequence of front tracking solutions is achieved using a variant of the singular mapping technique invented by Temple [69]. The front tracking method has no CFL-condition associated with it, and it does not discriminate between stiff and non-stiff source terms. This makes it an attractive approach for stiff problems, as is demonstrated in numerical examples. In addition, the numerical examples show that the front tracking method is able to preserve steady-state solutions (or achieving them in the long time limit) with good accuracy.
\end{abstract}

\section{INTRODUCTION}

We present and analyze a robust front tracking method for computing entropy solutions to the Cauchy problem for scalar balance equations with form

$$
u_{t}+f(u)_{x}=k^{\prime}(x) g(u), \quad u(x, 0)=u_{0}(x), \quad(x, t) \in \Pi_{T},
$$

where $\Pi_{T}$ denotes the space-time cylinder $\mathbf{R} \times(0, T) ; u=u(x, t)$ is the unknown function; and $f, g, k, u_{0}$ are given functions. We assume that $f(u), g(u)$ are locally Lipschitz continuous; $k(x)$ is continuously differentiable; and $u_{0}(x)$ is of bounded total variation. These are the standing assumptions that we make throughout this paper (but additional assumptions will be introduced later on). Consequently, (1.1) admits a unique bounded entropy solution of bounded total variation [48]. A typical example is $k(x)=x$, so that (1.1) is a conservation law with a nonlinear source term $g(u)$, possibly stiff.

In recent years hyperbolic conservation laws with source terms (balance laws) such as (1.1) have been extensively studied from a numerical point of view. The reason for this is of course that such equations occur in a wide variety of applications, and standard shock capturing as well as front tracking schemes seem to fail to produce accurate solutions in certain important cases (see below). When devising numerical schemes for balance equations like (1.1) it is simple (and frequently done in the literature) to use operator splitting and solve the conservation law

$$
u_{t}+f(u)_{x}=0
$$

and the ordinary differential equation

$$
u_{t}=k^{\prime}(x) g(u)
$$

sequentially for some small time step $\Delta t$, using the result of one computation as the initial data for the other. In this way, one can use specialized methods for the conservation law and for the ordinary differential equation, see $[50,29,34,44,40]$ for some applications of this strategy in the context of front tracking methods. In some cases this approach works well (see Langseth, Tveito, and Winther [50] for a favorable error estimate for operator splitting), but may also give unacceptably large errors for reasonable choices of $\Delta t$. This is especially evident if $k^{\prime}$ is large, so

Date: February 24, 2003.

Key words and phrases. scalar conservation law, source term, entropy solution, front tracking, convergence.

This work has been supported in part by the BeMatA program of The Research Council of Norway and the European network HYKE, funded by the EC as contract HPRN-CT-2002-00282. K. H. Karlsen is grateful to Hermano Frid for discussions. 
that the ordinary differential equation is stiff (see LeVeque and Yee [52]), an exception being stiff source terms of dissipative nature as in Tang [68], see also Helzel, LeVeque, and Warnecke [28] for a modified splitting method for approximating detonation waves.

Unacceptable errors occur in operator splitting methods also when seeking to preserve steadystate solutions or reaching them in the long time limit. Smooth solutions of (1.1) will satisfy the quasilinear equation

$$
u_{t}+f^{\prime}(u) u_{x}=k^{\prime}(x) g(u),
$$

multiplying this equation by $f^{\prime} / g$ and rearranging we find that

or

$$
\frac{f^{\prime}(u)}{g(u)} u_{t}+f^{\prime}(u)\left(\frac{f^{\prime}(u)}{g(u)} u_{x}-k^{\prime}(x)\right)=0,
$$

$$
(\Phi(u)-k)_{t}+f^{\prime}(u)(\Phi(u)-k)_{x}=0,
$$

where $\Phi$ is a primitive of $f^{\prime} / g$. This shows that the quantity $\Phi-k$ is advected passively with a speed given by $f^{\prime}(u)$. Using a splitting scheme for (1.3) will not respect this advection for smooth (parts of) the solution. Moreover, a (smooth) steady-state solution of (1.1) obeys

$$
f(u)_{x}=k^{\prime}(x) g(u)
$$

which may be equivalently stated as

$$
(\Phi(u)-k(x))_{x}=0,
$$

that is $\Phi(u)-k$ should be constant. Standard splitting schemes do not respect (1.4), neither do standard shock-capturing schemes that are based on adding in the source term as a correction to the conservation law step.

Many authors have designed unsplit schemes based on the idea of modifying the Riemann problem to account for the source term in such a way that the steady-state equations are satisfied. Liu [54, 55] pioneered this idea of using a generalized Riemann problem in the context of quasione dimensional (area varying) gas flow. He used the generalized Riemann problems to build a random choice scheme based on "piecewise stationary data" instead of "piecewise constant data". The random choice scheme was then used to prove the global existence of solutions for the nonhomogeneous system of gas flow equations. Glaz and Liu [19] used the generalized random choice scheme for numerical calculations of transonic nozzle flow (including the case of nonlinear resonance). In their paper, they also discuss scalar conservation laws with source term. Glimm, Marshall, and Plohr [20] and van Leer [70] devised second order schemes for such gas flow using the idea of Liu. Isaacson and Temple [39] analyzed a Godunov scheme for a general class of (resonant) scalar balance equations using a generalized Riemann problem (see also the discussion about resonance later on in this introduction). Chen and Glimm [10] demonstrated the existence of global solutions to the Euler equations of compressible isentropic gas dynamics with geometric structure. They did so by proving convergence of a Godunov scheme with a generalized Riemann solver (in the spirit of Liu) using steady-state solutions to incorporate the geometrical source term. Greenberg and Le Roux [25] (see also [24, 21, 26]) introduced the so-called well-balanced Godunov schemes for scalar equations. These schemes are also based on appropriately modifying the Riemann problem to account for the source term in such a way that the delicate balance in (1.4) is respected. The well-balanced schemes have been extended to systems by Gosse in [22, 23]. Another (but related) Godunov scheme can be found in LeVeque [51], which is able to capture the steady-state relation (1.4) by building it into the flux calculations. The balance (1.4) is achieved in his quasi-steady method by introducing additional Riemann problems at the cell center of each grid cell whose flux difference cancel exactly the source term. A similar approach can be found in Jenny and Müller [41] and their so-called Rankine-Hugoniot Riemann solver. In Zhou, Causon, Mingham, and Ingram[73], the authors derive a well-balanced scheme for the shallow water equations with bottom topography, which is based on the Harten-Lax-van Leer (HLL) approximate Riemann solver and the surface gradient method. For the shallow water system, Gallouët, Hérard, and Seguin [15] present a well-balanced Godunov scheme based on a very simple (linearized) approximate Riemann 
solver that preserves all steady-states. Kinetic equilibrium schemes (with reflections) are suggested by Botchorishvili, Perthame, and Vasseur [5] for scalar equations and by Perthame and Simeoni [61] for the shallow water equations with bottom topography. Gas-kinetic schemes that account for the source terms in the flux calculation across a cell interface are studied in $\mathrm{Xu}$ [72] and Tang, Tang, and Xu [67], again for the shallow water equations. Sweby [66] noted early that the popular TVD concept used to design high-resolution schemes for homogenous conservation laws is not appropriate for nonhomogeneous conservation laws, and he suggested to transform the nonhomogeneous problem to a homogeneous problem before applying a TVD scheme. Roe [65] suggested to apply high-resolution upwind schemes to a modified flux that includes the source term ("upwinding of the source term"). In the same spirit, Bermúdez and Vazquez [4] (see also $[3,71,37,16,9,59,60]$ for related papers) extended some upwind (flux-difference splitting and flux-vector splitting) schemes to hyperbolic systems of conservation laws with source terms (shallow water equations). To ensure that the numerical schemes approximate to high order steady-state solutions, they introduced the so-called "conservation property" (this property can be seen related to Liu's "piecewise stationary discretization" as well as LeRoux and Greenberg's notion of well-balanced schemes [23]). In [23] it was proved that the extensions of the Q-schemes of van Leer and Roe satisfy the conservation property but that the extensions of the flux-vector splitting schemes do not. Another simple method for processing source terms so that (1.4) is respected with high accuracy can be found in Jin [42]. His approach applies to Godunov and Roe type upwind schemes, but does not require any modification of the Riemann solver, since he is using the interface values instead of the cell-averages to process the source. Central-upwind schemes with a good treatment of the source terms in the shallow water equations can be found in Kurganov and Levy [49].

The goal of this paper is to devise a front tracking method for the scalar balance equation (1.1) which is able to cope with stiff sources as well as capturing steady-state solutions (1.4). The starting point is to write (1.1) as a quasilinear system of two equations, by adding the trivial equation $k_{t}=0$, which gives

$$
U_{t}+A(U) U_{x}=0, \quad U=\left(\begin{array}{l}
u \\
k
\end{array}\right), \quad A(U)=\left(\begin{array}{cc}
f^{\prime}(u) & -g(u) \\
0 & 0
\end{array}\right),
$$

and then (roughly speaking) apply front tracking to this system. Hence the effect of the source term in (1.1) is taken into account in the Riemann solver (no need to employ operator splitting).

The characteristic speeds of the system (1.5) are $\lambda_{1}=f^{\prime}(u)$ and $\lambda_{2}=0$. If $f^{\prime}(u)=0$ for some $u$ then the eigenvalues coincide, and we have so-called resonance (see, e.g., $[55,19,56,38,39])$. For such resonant systems, one can demonstrate that the total variation of the approximate solutions generated by the Glimm scheme or front tracking is not necessarily uniformly bounded, hence showing that such schemes converge is more complicated and does not follow the standard route, see, e.g., Temple [69], Gimse and Risebro [18], and Klingenberg and Risebro [47].

The suggested front tracking method is a numerical scheme where the solution of the Riemann problem to (1.5) is approximated by a piecewise constant (in $x / t$ ) function, whose discontinuities we call fronts. Since we have finite speed of propagation, the fronts will interact at some strictly positive time $t_{1}$, at this time we solve the resulting Riemann problem approximately, again giving fronts which we can propagate further in time. By studying interactions of fronts, we show that this gives a globally defined function $u^{h}(x, t)$, where $h$ is a parameter controlling the accuracy of the approximate Riemann solution. We show also that the front tracking method produces a sequence of approximations $\left\{u^{h}\right\}_{h>0}$ that converges to the unique entropy solution of (1.1). As alluded to above, although the solution that we seek to approximate has bounded total variation, it is not easy (if possible) to show that the total variation of $u^{h}$ is uniformly bounded (in $h$ ). Instead we shall utilize the singular mapping technique, first used by Temple [69] to analyze the polymer system, and later used by several authors to analyze conservation laws with a $x$ - discontinuous flux function (see, e.g., [39, 18, 47]), to show that the sequence of approximate solutions generated by front tracking is compact.

The core of the singular mapping approach is that, by (1.3), the total variation of $\Phi\left(u^{h}\right)-k^{h}$ ought to be bounded (here $k^{h}$ denotes a piecewise constant approximation to $k(x)$ ). However, since 
$f^{\prime}$ can vanish, the mapping $u \mapsto \Phi(u)$ in not invertible. Instead of $\Phi$, we consider the invertible mapping

$$
u \mapsto z(u)=\int^{u}\left|\frac{f^{\prime}(\xi)}{g(\xi)}\right| d \xi,
$$

and show that the total variation of $z^{h}:=z\left(u^{h}\right)$ is bounded independently of $h$. In turn this implies that the sequence $\left\{z^{h}\right\}_{h>0}$ is compact in $L^{1}$. If $\bar{z}$ is a limit of $z^{h}$, then we define

$$
u=z^{-1}(\bar{z}),
$$

and show that $u$ is an entropy solution of (1.1) in the sense of Kružkov [48].

Our analysis uses interaction estimates, and is therefore heavily dependent on the structure of the solution of the Riemann problem for (1.5). This solution is of course again dependent on the particular form of $f$ and $g$. Regarding the flux $f$, we assume that

$$
\left\{\begin{array}{l}
\text { there exists a unique value } u^{*} \text { such that } \\
f^{\prime}(u)>0 \text { for } u<u^{*} \text { and } f^{\prime}(u)<0 \text { for } u>u^{*} .
\end{array}\right.
$$

Regarding the source $g$, we assume that

$$
\left\{\begin{array}{l}
\text { there exists a value } u_{*}<u^{*} \text { such that } \\
g\left(u_{*}\right)=0 \text { and } g(u)>0 \text { for } u>u_{*} .
\end{array}\right.
$$

Furthermore, we shall demand that the initial data $u_{0}(x)$ take values in the interval $\left(u_{*}, M\right)$ for some finite constant $M$ :

$$
u_{0}(x) \in\left(u_{*}, M\right), \quad \forall x \in \mathbf{R} .
$$

For the nonresonant (or strictly hyperbolic) case, Amadori, Gosse, and Guerra [2] established recently the existence and uniqueness of $B V$ solutions to the Cauchy problem for hyperbolic systems of equations. Their existence proof was based on front tracking (different from ours), but in the nonresonant case the solution of the Riemann problem is in some sense simpler, since the waves arising from discontinuities in the source do not interfere with the "usual" waves. We also mention that Hong [36] has established existence of a solution via the Glimm scheme.

Very few of the papers cited above on numerical processing of source terms deal with the resonant case (as we do herein). However, numerical schemes for resonant scalar balance equations were considered by Noussair [57], in which he showed that the Glimm scheme and the Godunov scheme converged to weak solutions by using the singular mapping technique. His work was inspired by Isaacson and Temple [39], who proved convergence of the Godunov scheme for a particular class of resonant scalar balance equations. Although there are no convergence results yet for resonant (non-strictly hyperbolic) systems of balance equations, there are some papers [1, 58] that present (exact) Riemann solvers for the shallow water equations with bottom topography. These exact Riemann solvers can be used to build Godunov and front tracking methods for shallow water equations with a possibly discontinuous bottom topography. Proving that these methods converge remains an interesting open problem.

At the end of this introduction is seems appropriate to put the front tracking method into a historical perspective. Front tracking for scalar conservation laws (without source term) was introduced by Dafermos [11] as a mathematical tool for constructing and deriving properties of entropy solutions. It was later proved by Holden, Holden, and Høegh-Krohn [30, 31] that Dafermos' construction procedure was well-defined and could be viewed as a convergent numerical method. Front tracking was extended to systems of equations by Bressan [7], DiPerna [12], and Risebro [62], who used the method to give an alternative proof of Glimm's famous existence result for hyperbolic systems. Recently a modification of the front tracking method was used by Bressan and his collaborators to prove stability and uniqueness of weak solutions of strictly hyperbolic systems of conservation laws [8]. The front tracking method was used by Risebro and Tveito $[63,64]$ to numerically solve the Euler equations of gas dynamics and a non-strictly hyperbolic system modeling polymer flow. Holden and Risebro [33] extended the scalar front tracking method to multi-dimensional scalar conservation laws by means of dimensional splitting, see also [43]. It 
should be noted that no $\mathrm{CFL}$-condition is associated with the front tracking method, which implies that the method is fast compared with conventional difference schemes. Multi-dimensional scalar computations using CFL-numbers as high as $10-20$ have been reported, see Lie et al. [53]. Computational results for multi-dimensional systems (using CFL-numbers $2-5$ ) can be found in Holden et al. [32, 29] and Haugse, Karlsen, Lie, and Natvig [27]. A front tracking method for conservation laws with discontinuous coefficients is devised and analyzed in Gimse and Risebro [18] and Klingenberg and Risebro [47] (to mention only a few relevant references). Front tracking for Hamilton-Jacobi equations is the topic in Karlsen and Risebro [45, 46]. For front tracking applied to viscous conservation laws, see [14] and the references cited therein. An oil reservoir simulator based on the front tracking idea is described in [6]. We refer to the book [35] by Holden and Risebro for a general introduction to front tracking methods.

The rest of this paper is organized as follows, in Section 2 we review the solution of the Riemann problem and show that the front tracking approximation is well defined. In Section 3 we establish the compactness hinted at above, and show that the sequence of front tracking solutions converges to the unique entropy solution of (1.1). In Section 4 we present three numerical examples which demonstrate how the front tracking scheme is independent of the stiffness of the source term and moreover is able to capture steady-state solutions. We summarize our findings in Section 5.

\section{THE FRONT TRACKING METHOD}

In this section we present our front tracking method and prove also that it is well-defined.

2.1. The Riemann problem. We seek the solution of the Riemann problem

$$
u_{t}+f(u)_{x}=\left(a_{r}-a_{l}\right) \delta(x) g(u), \quad u(x, 0)= \begin{cases}u_{l} & x<0 \\ u_{r} & x \geq 0\end{cases}
$$

where $\delta(x)$ denotes the unit Dirac mass located at $x=0$. By solving the Riemann problem for (2.1), we really mean solving the problem

$$
U_{t}+A(U) U_{x}=0, \quad U=\left(\begin{array}{c}
u \\
a
\end{array}\right), \quad U(x, 0)= \begin{cases}U_{l}=\left(u_{l}, a_{l}\right), & x<0 \\
U_{r}=\left(u_{r}, a_{r}\right), & x>0\end{cases}
$$

where the matrix $A(U)$ is defined in (1.5). We recall that the characteristic speeds of the above system are $\lambda_{1}=f^{\prime}(u)$ and $\lambda_{2}=0$.

Observe that a non-conservative product occurs in (2.1), so that the solution of the Riemann problem becomes ambagious. But the special structure of the problem at hand makes it possible to construct a unique self-similar solution of the Riemann problem (2.1), which consists of waves that obey the Kružkov entropy condition [48] to the left and right of $x=0$ and a standing wave (contact discontinuity) sitting at $x=0$ satisfying the additional condition (compare with (1.3))

$$
\Phi(u(0-, t))-a_{l}=\Phi(u(0+, t))-a_{r},
$$

where

$$
\Phi(u)=\int^{u} \frac{f^{\prime}(\xi)}{g(\xi)} d \xi
$$

see $[39,57]$. We refer to Gosse $[22,23]$ for the simpler nonresonant case $f^{\prime} \neq 0$. He uses a framework of non-conservative products to make sense to the solution of the Riemann problem.

We next detail the solution of the Riemann problem $(2.1)$ (here we follow $[39,57,17]$ closely). Our prototype for this problem is the case where

$$
f(u)=4 u(1-u) \text { and } g(u)=u,
$$

so that $u_{*}$ in (1.7) is zero and $u^{*}$ in (1.6) is $1 / 2$. In this case we assume that $u_{l}$ and $u_{r}$ are positive, since for our prototype problem $\Phi$ will be given by

$$
\Phi(u)=4 \ln \left(u e^{-2 u}\right), \quad u>0 .
$$




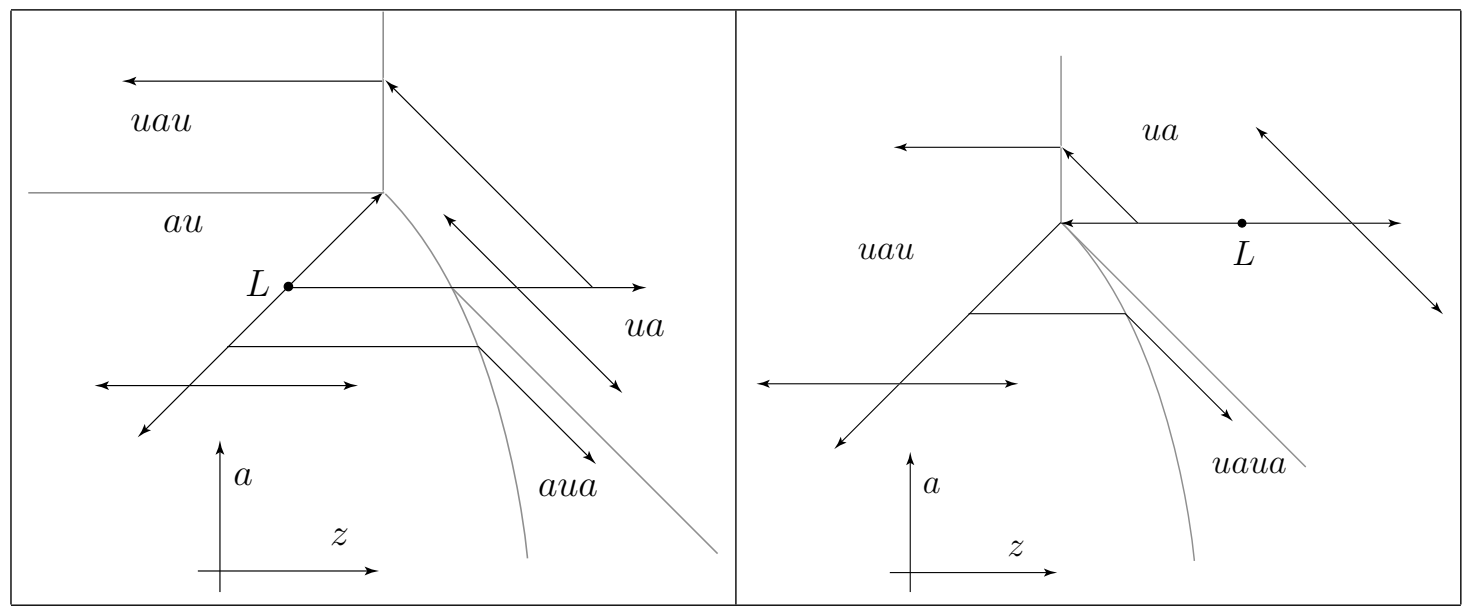

FiguRE 1. The solution of the Riemann problem (2.1). Left: $z_{l} \leq 0$. Right: $z_{l} \geq 0$.

The Riemann problem will be solved in terms of $a$-waves, which are waves with speed $\lambda_{2}=0$ over which

$$
\Phi(u)+a
$$

is constant, and $u$-waves with speed $\lambda_{1}=f^{\prime}(u)$, over which $a$ is constant. Define the singular mapping $u \mapsto z(u)$ by

$$
z(u)=\operatorname{sign}\left(u-u^{*}\right)\left(\Phi(u)-\Phi\left(u^{*}\right)\right) .
$$

For our prototype problem $(2.4), z(u)$ is defined for $u>0$, and in the general case for $u>u_{*}$. For later use, we state the following properties of the singular mapping:

Lemma 2.1. The singular mapping $u \mapsto z(u)$ is a strictly increasing continuous function on $\left(u_{*}, \infty\right)$, with a continuous inverse $z^{-1}(\cdot)$.

Proof. The proof is easy and left to the reader.

Following [47], it is advantageous to view the solution in $(z, a)$ coordinates rather than $(u, a)$ coordinates, since in this case the $a$-waves will be straight lines with slope \pm 1 . The solution of the Riemann problem is indicated in Figure 1. There are basically two different cases depending on whether the left state (in the figure labeled $L)\left(z_{l}, a_{l}\right)$ has $z_{l}$ positive or negative. To find a particular solution, follow the arrows from $L$ to any desired right state $\left(z_{r}, a_{r}\right)$. If $z_{l} \leq 0$, then the $(z, a)$ plane is divided into 4 regions as follows. Let $u_{0}(u)$ (not to be confused with the initial function) be defined for $u \leq u^{*}$ as the unique point such that

$$
f\left(u_{0}(u)\right)=f(u), \quad \text { and } \quad u_{0}(u) \geq u^{*} .
$$

Then set $\sigma_{0}(a)$ to be the curve defined for $a \leq a_{l}-z_{l}$ by

$$
\sigma_{0}(a)=z\left(u_{0}\left(z^{-1}\left(a-\left(a_{l}-z_{l}\right)\right)\right)\right),
$$

and set $\sigma_{0}(a)=0$ for $a>a_{l}-z_{l}$. Now $\sigma_{0}$ is defined so that a point on the $a$-wave curve through $\left(z_{l}, a_{l}\right)$, given by $z=a-\left(a_{l}-z_{l}\right)$ for $a \leq a_{l}-z_{l}$, can be connected to the point $\left(\sigma_{0}(a), a\right)$ by a $u$-wave of zero speed. In Figure $1 \sigma_{0}$ is the curved line. Next, divide the $(z, a)$ plane into the four regions:

$$
\begin{aligned}
L_{a u} & =\left\{(z, a) \mid a \leq a_{l}-z_{l} \text { and } z \leq \sigma_{0}(a)\right\}, \\
L_{a u a} & =\left\{(z, a) \mid a \leq a_{l}+\sigma_{0}\left(a_{l}\right)-z \text { and } z>\sigma_{0}(a)\right\}, \\
L_{u a} & =\left\{(z, a) \mid a>a_{l}+\sigma_{0}\left(a_{l}\right)-z \text { and } z>\sigma_{0}(a)\right\}, \\
L_{\text {uau }} & =\left\{(z, a) \mid a>a_{l}-z_{l}, \quad \text { and } z \leq 0\right\} .
\end{aligned}
$$


From the left part of Figure 1 the gray lines mark the boundaries between these regions. To find a particular solution, we first determine a region $L_{X}$ such that $\left(z_{r}, a_{r}\right) \in L_{X}$. Then $X$ gives the wave pattern with which the Riemann problem is solved.

As an example, assume that $\left(z_{r}, a_{r}\right) \in L_{u a u}$. In this case the Riemann is solved by a $u$-wave from $\left(z_{l}, a_{l}\right)$ to $\left(a_{r}-a_{l}, a_{l}\right)$ followed by an $a$-wave to $\left(0, a_{r}\right)$ and finally by a $u$-wave to $\left(z_{r}, a_{r}\right)$. By our assumptions on $f$, the first $u$-wave will be a shock, and the second $u$-wave will be a rarefaction.

If $\left(z_{r}, a_{r}\right) \in L_{a u a}$ the solution is given by an $a$-wave from $\left(z_{l}, a_{l}\right)$ to $\left(a_{l}-z_{l}-a_{m}, a_{m}\right)$ followed by a $u$-wave (of speed zero) to $\left(a_{r}+z_{r}-a_{m}, a_{m}\right)$ and then by an $a$ wave to $\left(z_{r}, a_{r}\right)$, where $a_{m}$ denotes the unique solution of

$$
a_{m}+\sigma_{0}\left(a_{m}\right)=a_{r}+z_{r} .
$$

Note that in this case the intermediate states $\left(a_{l}-z_{l}-a_{m}, a_{m}\right)$ and $\left(a_{r}+z_{r}-a_{m}, a_{m}\right)$ will not appear in $(x, t)$ space since all waves in the aua combination have zero speed. The solutions in the other two cases, $a u$ and $u a$, are now obvious.

If $z_{l}>0$, we consult the right part of Figure 1 to find the solution. Now, the $(z, a)$ plane is divided into the three regions

$$
\begin{aligned}
R_{u a} & =\left\{(z, a) \mid a>a_{l}-z \text { and } z \geq 0\right\}, \\
R_{\text {uau }} & =\left\{(z, a) \mid z<\sigma_{0}(a)\right\}, \\
R_{\text {uaua }} & =\left\{(z, a) \mid z \geq \sigma_{0}(a) \text { and } a \leq a_{l}-z\right\} .
\end{aligned}
$$

Similarly to the previous case, we have that if $\left(z_{r}, a_{r}\right) \in R_{X}$, then the solution to the Riemann problem consists of the wave pattern $X$, and is found by following the arrows from $\left(z_{l}, a_{l}\right)$ to $\left(z_{r}, a_{r}\right)$ using this wave pattern. Again, note that if we have a solution with the wave pattern uaua, then the intermediate states to the right of the first $a$-wave and to the left of the last $a$-wave will not be visible in $(x, t)$.

2.2. The numerical algorithm. Equipped with a Riemann solver, we can now present the front tracking algorithm for constructing approximations to the solution of the initial value problem

$$
u_{t}+f(u)_{x}=k^{\prime}(x) g(u), \quad u(x, 0)=u_{0}(x) .
$$

The front tracking algorithm is inspired by one used in $[18,47]$ to analyze scalar conservation laws with a $x$ - discontinuous flux function.

Fix a (small) number $h>0$ and let $(z, a)$ be as before. Then we define a grid in the $(z, a)$ plane with grid spacing $h$ by $z_{i}=i h, a_{j}=j h$ for $i$ and $j$ in $\mathbf{Z}$. Let $f^{h}$ denote the piecewise linear approximation to $f$ made by linearly interpolating between the points

$$
u_{i}=z^{-1}\left(z_{i}\right) \quad \text { and } \quad u_{i+1}=z^{-1}\left(z_{i+1}\right), \quad i=\ldots,-2,-1,0,1,2, \ldots
$$

where $z(\cdot)$ is defined in $(2.5)$, i.e.,

$$
f^{h}(u)=f_{i}+\left(u-u_{i}\right) \frac{f_{i+1}-f_{i}}{u_{i+1}-u_{i}}, \quad \text { for } u \in\left[u_{i}, u_{i+1}\right],
$$

where $f_{i}=f\left(u_{i}\right)$. Then we approximate $k(x)$ by a piecewise constant function $k^{h}(x)$ taking values in the set $\left\{a_{j}\right\}_{j \in \mathbf{Z}}$. Therefore we can write

$$
\begin{aligned}
k^{h}(x) & =\sum_{i}\left[a_{j_{i+1}}-a_{j_{i}}\right] H\left(x-x_{i}\right), \\
\left(k^{h}\right)^{\prime}(x) & =\sum_{i}\left[a_{j_{i+1}}-a_{j_{i}}\right] \delta\left(x-x_{i}\right),
\end{aligned}
$$

where $H(x)$ is the Heaviside function with a jump at $x=0$ and $\delta(x)$ is the unit Dirac mass located at $x=0$. Finally we approximate the initial function $u_{0}(x)$ by a piecewise constant function $u_{0}^{h}$ taking values in the set $\left\{u_{i}\right\}_{i \in \mathbf{Z}}$. Then each discontinuity point of $u_{0}^{h}$ and of $k^{h}$ determines a Riemann problem. Note that discontinuity points of $k^{h}$ can also be discontinuity points of $u_{0}^{h}$. By 
construction of $k^{h}$ and $u_{0}^{h}$ the left and right states of these Riemann problems will be on the grid in the $(z, a)$ plane. Thus for a given $i$, the relevant Riemann problem reads

$$
u_{t}+f(u)_{x}=\left[a_{r}-a_{l}\right] g(u) \delta\left(x-x_{i}\right), \quad u(x, 0)= \begin{cases}u_{l} & x<x_{i}, \\ u_{r} & x \geq x_{i}\end{cases}
$$

where $a_{l}=j_{l} h, a_{r}=j_{r} h, z\left(u_{l}\right)=i_{l} h$ and $z\left(u_{r}\right)=i_{r} h$ for certain integers $i_{l}, i_{r}, j_{l}$, and $j_{r}$. Next, observe that in the $(x, t)$ plane, the solution of this Riemann problem will have intermediate values that are also grid points. Discontinuity points of $u_{0}^{h}$ that are not discontinuity points of $k^{h}$ determine Riemann problems for the scalar equation

$$
u_{t}+f^{h}(u)_{x}=0, \quad u(x, 0)= \begin{cases}u_{i_{l}} & x<\bar{x} \\ u_{i_{r}} & x \geq \bar{x}\end{cases}
$$

where $\bar{x}$ denotes a discontinuity point of $k^{h}$. By the construction of $f^{h}$, the solution of this Riemann problem will be a piecewise constant of $(x-\bar{x}) / t$ and take values in the set $\left\{u_{i}\right\}_{i=i_{l}}^{i=i_{r}}$. In the case where $f^{\prime \prime}<0$, if $i_{l} \leq i_{r}$, the solution reads

$$
u(x, t)= \begin{cases}u_{i_{l}} & (x-\bar{x})<s\left(i_{l}, i_{r}\right) t \\ u_{i_{r}} & (x-\bar{x}) \geq s\left(i_{l}, i_{r}\right) t\end{cases}
$$

where $s(i, j)=\left(f_{i}-f_{j}\right) /\left(u_{i}-u_{j}\right)$. If $i_{l}>i_{r}$ the solution consists of the approximate rarefaction wave

$$
u(x, t)= \begin{cases}u_{i_{l}} & (x-\bar{x})<s\left(i_{l}-1, i_{l}\right) t, \\ u_{j} & s(j-1, j) t \leq(x-\bar{x})<s(j, j+1) t \quad \text { for } j=i_{l}, \ldots, i_{r}, \\ u_{i_{r}} & s\left(i_{r}, i_{r}+1\right) t \leq(x-\bar{x}) .\end{cases}
$$

Now we are in a position to define the front tracking algorithm. Start by solving the Riemann problems defined by $k^{h}$ and $u_{0}^{h}$. At discontinuities of $k^{h}$ we solve the Riemann problem (2.10), but using $f^{h}$ instead of $f$ when computing the $u$ waves. This will give a piecewise constant function taking values from the grid points. At the discontinuity point of $u_{0}^{h}$ we solve the Riemann problems defined by (2.11). Since all waves have finite speed of propagation, for small $t$, this will give a piecewise constant function in $x$. We denote this function by $u^{h}(x, t)$. So far, $u^{h}$ is defined for $t \leq t_{1}$, where $t_{1}$ is the first time when discontinuities in $u^{h}$, hereafter called fronts, collide. However, at $t=t_{1}$, the situation is similar to the initial situation, and therefore we can "solve" the Riemann problems defined by $u^{h}\left(\cdot, t_{1}\right)$ and $k^{h}$, and therefore define $u^{h}$ until the next collision of fronts. Computationally, we are actually in a much better situation at $t=t_{1}$ than at $t=0$, since we have already solved the Riemann problems for those fronts not colliding at $t_{1}$. It is clear that this process can be continued for an arbitrary number of collisions.

Although we can continue this process for as many collisions as we like, it is not yet clear whether there may be an infinite number of collisions in finite time. If this was the case then $u^{h}$ could not be defined past this buildup of collision times. Hence we must examine the algorithm more closely.

2.3. The numerical algorithm is well-defined. We shall need to define the strength of the fronts in the front tracking solution $u^{h}$. First we note that $u^{h}$ can be seen as a sequence of fronts, each of type $u$ or $a$. A front separates states $\left(z_{l}, a_{l}\right)$ and $\left(z_{r}, a_{r}\right)$.

We start by defining the strength of $u$ fronts, denoted by $F(u)$, by

$$
F(u)=\left|z_{l}-z_{r}\right| \text {. }
$$

The strength of $a$ fronts, denoted by $F(a)$, is more complicated to define:

$$
F(a)=\left\{\begin{array}{ll}
\left\{\begin{array}{ll}
4\left|a_{l}-a_{r}\right| & \text { if } a_{r}<a_{l}, \\
2\left|a_{l}-a_{r}\right| & \text { if } a_{r}>a_{l},
\end{array} \quad \text { if } z_{l} \geq 0 \text { and } z_{r} \geq 0\right. \\
4\left|a_{l}-a_{r}\right| & \text { if } a_{r}>a_{l}, \\
2\left|a_{l}-a_{r}\right| & \text { if } a_{r}<a_{l},
\end{array} \quad \text { if } z_{l} \leq 0 \text { and } z_{r} \leq 0 .\right.
$$




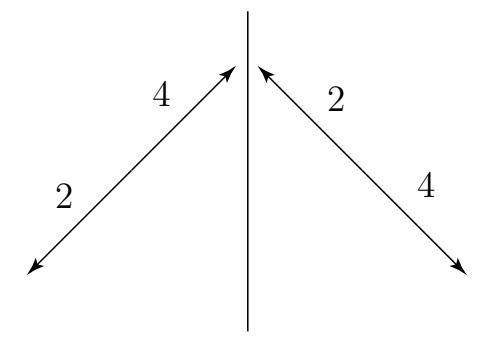

FigurE 2. Schematic representation of the definition of $F(a)$.

See Figure 2 for an illustration of how $F$ is defined for $a$ fronts. To read this figure, a wave going in the direction of the indicated arrows is given the weight displayed along the relevant arrow. $(\nearrow=4, \swarrow=2, \nwarrow=2, \searrow=4, \leftrightarrow=1)$. For a sequence of fronts

$$
w=w_{1} w_{2} w_{3} \cdots w_{N}
$$

we define $F$ additively by

$$
F(w)=\sum_{i=1}^{N} F\left(w_{i}\right) .
$$

Since $u^{h}$ can be seen as a sequence of fronts, $F\left(u^{h}\right)$ is defined in the obvious way.

Now we can state the following crucial lemma (which will be used also in Section 3 to derive compactness of a sequence of front tracking solutions):

Lemma 2.2. Let $w=w_{1} w_{2} \cdots w_{N}$ be any sequence of fronts such that the left state of $w_{1}$ is $\left(z_{l}, a_{l}\right)$ and the right state of $w_{N}$ is $\left(z_{r}, a_{r}\right)$, and that $w$ defines a continuous path in the $(z, a)$ plane. Let $\left[\left(z_{l}, a_{l}\right),\left(z_{r}, a_{r}\right)\right]$ denote the wave path defined by the solution of the Riemann problem (2.1). Then

$$
F\left(\left[\left(z_{l}, a_{l}\right),\left(z_{r}, a_{r}\right)\right]\right) \leq F(w) .
$$

Proof. Since the structure of the Riemann problem studied here is very similar to the Riemann problems studied in $[47,69,39]$, we only detail the case in which our Riemann problem has a different wave structure than in these works, namely the wave patterns aua and uaua.

First we assume that the Riemann problem is solved by a aua combination. In this case we can find a (unique) wave pattern $\bar{a} \bar{u}$ connecting the left and the right states such that $F($ Riemann solution $)=F(\bar{a} \bar{u})$. Since the Riemann solution reported in [47] is (in the notation used here) $a u$, the lemma follows in this case.

Similarly, if the Riemann solution is given by uaua, we can replace the three last waves by a $\bar{a} \bar{u}$ combination such that $F(u a u a)=F(u \bar{a} \bar{u})$. As before, the Riemann solution in [47] is $u \bar{a} \bar{u}$, and the lemma holds in this case. Therefore the lemma will also hold in our case.

To show that the front tracking construction is well-defined, and that we can define the function $u^{h}(\cdot, t)$ for any $t>0$, we must examine in some detail what happens when fronts interact.

We start by considering interaction of stationary aua-fronts with moving $u$-fronts. First assume that the $u$-front is colliding with the aua-front from the right. In particular the speed of the $u$-front must be negative, and the aua front connects a state $\left(z_{l}, a_{l}\right)$ with a state $\left(z_{m}, a_{m}\right)$. Then the front connecting $\left(z_{l}, a_{l}\right)$ and $\left(z_{r}, a_{r}\right)$ must be a single discontinuity, and since $f^{\prime \prime}<0$, we must have

$$
z_{r} \geq z_{l}-h \geq 0 \text {. }
$$

From Figure 1 we can see that the Riemann problem defined by $\left(z_{l}, a_{l}\right)$ and $\left(z_{r}, a_{r}\right)$ is solved by either a

Case 1 aua combination,

Case 2 ua combination, or

Case 3 au combination. 
In Case 1 or Case 2, the interaction leaves $F$ unchanged, and in Case 3, $F$ decreases by at least $2 h$. Note that in Case 3 , the $u$-front is "reflected".

Next, assume that a $u$-front of positive speed is colliding with the stationary aua-front. In this case, the aua-front connects states $\left(z_{m}, a_{m}\right)$ and $\left(z_{r}, a_{r}\right)$, and we have that

$$
z_{l} \leq z_{m}+h
$$

and if $z_{m}=0$, then $z_{l}<z_{m}$. From Figure 1 we observe that there are two cases regarding the solution of the Riemann problem defined by $\left(z_{l}, a_{l}\right)$ and $\left(z_{r}, a_{r}\right)$ :

Case 4 an aua combination, or

Case 5 an au combination.

Both of these leave $F$ unchanged.

Next we examine the interaction of stationary $a$-fronts and $u$-fronts. First we treat the interaction of an $a$-front and a $u$-front colliding from the right. Assume first that $z_{m} \leq 0$, in this case

$$
z_{r}>\sigma_{0}\left(a_{r}\right)
$$

since the $u$-front has negative speed. Then the resulting Riemann problem is solved by either a

Case 6 ua combination, or

Case 7 aua combination.

Both of these leave $F$ unchanged. If $z_{m} \geq 0$, we have that

$$
z_{r} \geq z_{m}-h,
$$

and if $z_{m}=0$ then $z_{r}>z_{m}$. The resulting Riemann problem is solved by one of the following combinations:

Case 8 a $u$ a combination,

Case 9 an aua combination, or

Case 10 an au combination.

Case 10 can hold only if $z_{l}=0$, and $z_{r}=z_{m}-h$. In Case $8 F$ is unchanged by the collision, and in Case 9 and $10 F$ decreases by $2 h$.

Next we consider collisions between a stationary $a$ front and a $u$ front coming from the left (with positive speed). First we discuss the case where $z_{m} \leq 0$. Now

$$
z_{l} \leq z_{m}+h, \quad \text { if } z_{m}<0,
$$

and $z_{l}<z_{m}$ if $z_{m}=0$. Then the Riemann problem is solved by either a

Case 11 au combination, or

Case 12 ua combination.

Case 12 can hold only if $z_{l}=z_{m}+h$ and $z_{r}=0$, and in this case $F$ decreases by $2 h$. If Case 11 holds, then $F$ is unchanged.

Finally if $z_{m} \geq 0$, then

$$
z_{m}>\sigma_{0}\left(a_{l}\right)
$$

since the $u$-front has positive speed. Consulting Figure 1, we find that the solution of the Riemann problem consists of either

Case 13 an aua combination, or

Case 14 an au combination.

Both of these alternatives leave $F$ unchanged.

Next we observe that in collisions between two (or more) $u$ fronts the number of fronts will always decrease, since the solution of the resulting Riemann problem will consist of a single front. Furthermore, the speed of the resulting front will be between the speeds of the leftmost and rightmost of the colliding fronts. This is so since $f$ and $f^{h}$ are concave.

Now we have discussed all types of collisions that may occur between fronts in $u^{h}$ for $t>0$. First we observe that for a fixed $h$, the number of fronts remains finite, since any collision results in at most two fronts. Secondly, whenever a $u$-front is "reflected" as a result of a collision, $F$ 
decreases by at least $2 h$. Consequently, for a fixed $h$, this can happen at most a finite number of times if $F\left(u^{h}(\cdot, 0)\right)$ is finite.

From these observations, we see that since for a fixed $h, k^{h}$ is constant for $x$ outside a bounded interval, after some finite time, all $u$ fronts of non-zero speed will have passed through the stationary fronts located at the discontinuities of $k^{h}$. Hence after this finite time, there will only be collisions between $u$ fronts. Furthermore, since the number of fronts decrease for each such collision, there can be only a finite number of these collisions.

We summarize our discussion in the following lemma:

Lemma 2.3 (well-defined algorithm). For each discretization parameter $h>0$, there is only a finite number of collisions between fronts in the front tracking solution $u^{h}$ for any time $t>0$. In particular, $u^{h}$ can be defined in the whole region $(x, t) \in \mathbf{R} \times[0, \infty)$.

\section{Convergence analysis}

Now we set out to prove that the numerical method devised in Section 2 converges to the unique entropy solution of (1.1). We recall that solutions of (1.3) will not in general be differentiable, or even continuous, and thus we seek weak solutions $u \in L^{\infty}\left(\Pi_{T}\right)$ that satisfy

$$
\int_{0}^{T} \int_{\mathbf{R}} u \varphi_{t}+f(u) \varphi_{x}+k^{\prime}(x) g(u) \varphi d x d t+\int_{\mathbf{R}} u_{0}(x) \varphi(x, 0) d x=0,
$$

for all test functions $\varphi \in C_{0}^{\infty}(\mathbf{R} \times[0, T))$.

However, weak solutions are not unique, and the unique entropy solution to (1.3) is defined as the weak solution satisfying the Kružkov entropy condition [48]

$$
\begin{aligned}
\int_{0}^{T} \int_{\mathbf{R}}|u-c| \varphi_{t}+\operatorname{sign}(u-c)(f(u)-f(c)) \varphi_{x} & +\operatorname{sign}(u-c) k^{\prime}(x) g(u) \varphi d x d t \\
& +\int_{\mathbf{R}}\left|u_{0}-c\right| \varphi(x, 0) d x \geq 0,
\end{aligned}
$$

for all nonnegative $\varphi \in C_{0}^{\infty}(\mathbf{R} \times[0, T))$ and for all constants $c \in \mathbf{R}$. Actually, (3.2) implies (3.1). Here, $\operatorname{sign}(\cdot)$ the denotes the usual sign function with $\operatorname{sign}(0)=0$.

The results obtained in this section hold under the assumptions introduced in Section 1. Recall that the prototype problem that we have in mind is (2.4) with initial data $u_{0}$ taking positive values, which implies via the Riemann solution that the front tracking solution $u^{h}(\cdot, t)$ remains positive for all times $t>0$. More generally, we have the following desirable property:

Lemma 3.1 (lower bound on $\left.u^{h}\right)$. Let $u^{h}=u^{h}(x, t)$ be the front tracking solution defined in Section 2. Then

where $u_{*}$ is defined in (1.8).

$$
u^{h}(\cdot, t)>u_{*}, \quad \forall t>0,
$$

Our main convergence result (stated in Corollary 3.1) will be a consequence of Theorem 3.1 (compactness) and Theorem 3.2 (entropy satisfaction) found below.

Theorem 3.1 (compactness). Suppose $f, g, k, u_{0}$ satisfy the conditions stated in Section 1, and in particular (1.6), (1.7), and (1.8). Let $u^{h}=u^{h}(x, t)$ be the front tracking solution defined in Section 2. Then there exists a subsequence of $\left\{u^{h}\right\}_{h>0}$, still denoted by $\left\{u^{h}\right\}_{h>0}$, and a bounded function $u$ such that, as $h \rightarrow 0$,

$$
u^{h} \rightarrow u \quad \text { a.e. in } \Pi_{T} \text { and in } L_{\mathrm{loc}}^{1}\left(\Pi_{T}\right) .
$$

In fact, $u^{h} \rightarrow u$ in $C\left(0, T ; L_{\mathrm{loc}}^{1}(\mathbf{R})\right)$ and $u \in L^{\infty}\left(\Pi_{T}\right) \cap C\left(0, T ; L_{\mathrm{loc}}^{1}(\mathbf{R})\right)$.

Proof. Define the transformed approximate solution $z^{h}$ by

$$
z^{h}(x, t)=z\left(u^{h}(x, t)\right),
$$

where the singular mapping $u \mapsto z(u)$ is defined in (2.5) and it possesses the properties stated in Lemma 2.1. We shall prove that $\left\{z^{h}\right\}_{h>0}$ is compact in $L_{\mathrm{loc}}^{1}\left(\Pi_{T}\right)$. 
In terms of the functional $F$ defined in (2.14), we have the inequalities

$$
\left|z^{h}(\cdot, t)\right|_{B V(\mathbf{R})}+\left|k^{h}\right|_{B V(\mathbf{R})} \leq F\left(u^{h}(\cdot, t)\right) \leq 4\left(\left|z^{h}(\cdot, t)\right|_{B V(\mathbf{R})}+\left|k^{h}\right|_{B V(\mathbf{R})}\right), \quad t>0 .
$$

Observe that $F\left(u^{h}(\cdot, 0)\right)$ is bounded independently of $h$ since $\left|u_{0}\right|_{B V(\mathbf{R})}<\infty$. From this, (3.4), and Lemma 2.2, we have the uniform $B V$ bound

$$
\left|z^{h}(\cdot, t)\right|_{B V(\mathbf{R})} \leq C, \quad t>0
$$

for some constant $C$ that is independent of $h$. Hence $\left\{z^{h}(\cdot, t)\right\}$ is also bounded independently of $h$, and thus also $\left\{u^{h}\right\}$ is bounded. Let $U$ be a constant bounding $\left|u^{h}\right|$ :

$$
\left\|u^{h}\right\|_{L^{\infty}\left(\Pi_{T}\right)} \leq U
$$

By construction, the function $z^{h}(x, t)$ is piecewise constant in $x$, with discontinuities (fronts) moving with finite speed. Let $0=t_{0}<t_{1}<t_{2}<\cdots<t_{j}<\cdots<t_{M}$ be the collision times of $z^{h}$. Assume that $t_{j}<\tau_{1}<\tau_{2} \leq t_{j+1}$ for some $j \geq 0$. If we label the constant states of $z^{h}$ by $\left\{z_{\ell}\right\}$, then for $t \in\left(t_{j}, t_{j+1}\right]$

$$
z^{h}(x, t)=\sum_{\ell}\left(z_{\ell+1}-z_{\ell}\right) H\left(x-s_{\ell}\left(t-t_{j}\right)-x_{\ell}\right),
$$

where $s_{\ell}$ is the speed of the $\ell$ th discontinuity, and $x_{\ell}$ is the location of this discontinuity at $t_{j}+$. Interpreted as a measure,

$$
\partial_{t} z^{h}(x, t)=\sum_{\ell}\left(z_{\ell+1}-z_{\ell}\right) s_{\ell} \delta\left(x-s_{\ell}\left(t-t_{j}\right)-x_{\ell}\right) .
$$

Now we find

$$
\begin{aligned}
\int_{a}^{b}\left|z^{h}\left(x, \tau_{2}\right)-z^{h}\left(x, \tau_{1}\right)\right| d x & \leq \int_{a}^{b} \int_{\tau_{1}}^{\tau_{2}}\left|\partial_{t} z^{h}(x, t)\right| d t d x \\
& \leq \int_{\tau_{1}}^{\tau_{2}} \int_{\mathbf{R}} \sum_{\ell}\left|s_{\ell}\left(z_{\ell+1}-z_{\ell}\right)\right| \delta\left(x-s_{\ell}\left(t-t_{j}\right)-x_{\ell}\right) d x d t \\
& \leq\left(\tau_{2}-\tau_{1}\right) \max _{\ell}\left|s_{\ell}\right|\left|z^{h}\right|_{B V(\mathbf{R})},
\end{aligned}
$$

where $(a, b)$ is any bounded interval. Now letting $\tau_{1} \downarrow t_{j}$ and $\tau_{2} \uparrow t_{j+1}$, we find that

$$
\int_{a}^{b}\left|z^{h}\left(x, t_{j+1}\right)-z^{h}\left(x, t_{j}\right)\right| d x \leq C \max _{|u| \leq U}\left|f^{\prime}(u)\right|\left(t_{j+1}-t_{j}\right) .
$$

From this it follows that for any $t \geq s \geq 0$,

$$
\left\|z^{h}(\cdot, t)-z^{h}(\cdot, s)\right\|_{L^{1}((a, b))} \leq \max _{|u| \leq U}\left|f^{\prime}(u)\right| C(t-s) .
$$

for a constant $C$ that is independent of $h$. From standard theory (see, e.g., [35]), the uniform estimates (3.5) and (3.6) imply that there is a function $\bar{z}=\bar{z}(x, t)$ such that

$$
\bar{z} \in L^{\infty}\left(\Pi_{T}\right) \bigcap L^{\infty}\left(0, T ; L_{\mathrm{loc}}^{1}(\mathbf{R})\right),
$$

and a subsequence of $\left\{z^{h}\right\}$ (which we do not bother to relabel) such that $z^{h} \rightarrow \bar{z}$ strongly in $L_{\text {loc }}^{1}\left(\Pi_{T}\right)$. Actually we have more: $z^{h} \rightarrow \bar{z}$ in $C\left(0, T ; L_{\text {loc }}^{1}(\mathbf{R})\right)$ and $\bar{z} \in L^{\infty}\left(\Pi_{T}\right) \cap C\left(0, T ; L_{\text {loc }}^{1}(\mathbf{R})\right)$.

Since, by construction (3.3), we have

$$
u^{h}=z^{-1}\left(z^{h}\right)
$$

also $u^{h}$ will converge strongly in $L_{\text {loc }}^{1}\left(\Pi_{T}\right)$ and in $C\left(0, T ; L_{\text {loc }}^{1}(\mathbf{R})\right)$, thanks to Lemmas 2.1 and 3.1 . More precisely, we have

$$
u^{h} \rightarrow u, \quad \text { where } \quad u:=z^{-1}(\bar{z}) \in L^{\infty}\left(\Pi_{T}\right) \cap C\left(0, T ; L_{\text {loc }}^{1}(\mathbf{R})\right) .
$$

We shall need the following technical lemma. 
Lemma 3.2. Let $\Omega \subset \mathbf{R}^{d}$ be a bounded open set, $g \in L^{1}(\Omega)$, and suppose that $g_{\nu}(x) \rightarrow g(x)$ a.e. in $\Omega$. Then there exists a set $\Theta$, which is at most countable, such that for any $c \in \mathbf{R} \backslash \Theta$

$$
\operatorname{sign}\left(g_{\nu}(x)-c\right) \rightarrow \operatorname{sign}(g(x)-c) \quad \text { a.e. in } \Omega \text {. }
$$

Let $c \in \Theta$, and define

$$
\mathcal{E}_{c}=\{x \in \Omega \mid g(x)=c\} .
$$

It is possible to construct sequences $\left\{\underline{c}_{\nu}\right\}_{\nu=1}^{\infty},\left\{\bar{c}_{\nu}\right\}_{\nu=1}^{\infty}$ such that $\underline{c}_{\nu}, \bar{c}_{\nu} \in \mathbf{R} \backslash \Theta$ and

$$
\begin{aligned}
& \left\{\begin{array}{l}
\underline{c}_{\nu}<c, \quad \underline{c}_{\nu} \uparrow c \text { as } \nu \rightarrow \infty, \\
\operatorname{sign}\left(g(x)-\underline{c}_{\nu}\right) \rightarrow \operatorname{sign}(g(x)-c) \text { as } \nu \rightarrow \infty \text { for a.e. } x \in \Omega \backslash \mathcal{E}_{c},
\end{array}\right. \\
& \left\{\begin{array}{l}
\bar{c}_{\nu}>c, \quad \bar{c}_{\nu} \downarrow c \text { as } \nu \rightarrow \infty, \\
\operatorname{sign}\left(g(x)-\bar{c}_{\nu}\right) \rightarrow \operatorname{sign}(g(x)-c) \text { as } \nu \rightarrow \infty \text { for a.e. } x \in \Omega \backslash \mathcal{E}_{c} .
\end{array}\right.
\end{aligned}
$$

Proof. Fix $c \in \mathbf{R}$ and a point $x \in \Omega$ where $g_{\nu}(x) \rightarrow g(x)$, and $g(x) \neq c$. For $\nu$ sufficiently large, $\operatorname{sign}\left(g_{\nu}(x)-c\right)=\operatorname{sign}(g(x)-c)$, i.e., $\operatorname{sign}\left(g_{\nu}(x)-c\right)$ is constant and so converges to $\operatorname{sign}(g(x)-c)$. We have shown that for each $c \in \mathbf{R}, \operatorname{sign}\left(g_{\nu}-c\right) \rightarrow \operatorname{sign}(g-c)$ a.e. in $\Omega \backslash \mathcal{E}_{c}$. The proof will be done if we can show that all but at most countably many of the sets $\mathcal{E}_{c}$ have measure meas $\left(\mathcal{E}_{c}\right)=0$. To this end, define

$$
\mathcal{C}_{n}=\left\{c \in \mathbf{R} \mid \operatorname{meas}\left(\mathcal{E}_{c}\right) \geq \frac{1}{n}\right\} .
$$

Since $\Omega$ is bounded, $\mathcal{C}_{n}$ can only contain a finite number of points. Then

$$
\left\{c \in \mathbf{R} \mid \operatorname{meas}\left(\mathcal{E}_{c}\right)>0\right\}=\bigcup_{n>0} \mathcal{C}_{n}
$$

is an at most countable set.

For the first assertion in (3.7), fix $c \in \Theta$. It is clear that since $\Theta$ is at most countable, we can construct a sequence $\left\{\underline{c}_{\nu}\right\}_{\nu=1}^{\infty}$ such that $\underline{c}_{\nu}<c$ and $\underline{c}_{\nu} \rightarrow c$ as $\nu \rightarrow \infty$. Let $x \in \Omega \backslash \mathcal{E}_{c}$. Since $g(x) \neq c, \operatorname{sign}\left(g(x)-\underline{c}_{\nu}\right)=\operatorname{sign}(g(x)-c)$ for $\nu$ sufficiently large, and thus sign $\left(g(x)-\underline{c}_{\nu}\right)$ converges to sign $(g(x)-c)$. The second assertion in (3.7) is proven in a similar way.

We can now show that the limit function $u$ is a Kružkov entropy solution.

Theorem 3.2 (entropy satisfaction). Suppose $f, g, k, u_{0}$ satisfy the conditions stated in Section 1, and in particular (1.6), (1.7), and (1.8). Let $u^{h}=u^{h}(x, t)$ be the front tracking solution defined in Section 2. Let $u$ be a limit point (in the sense of Theorem 3.1) of $\left\{u^{h}\right\}_{h>0}$. Then u satisfies the Kružkov entropy condition (3.2).

Proof. We need to show that (3.2) holds for the front tracking limit function $u$. To simplify the presentation, we employ the notations (suppressing the $c$ dependency)

$$
\eta(u)=|u-c| \quad \text { and } \quad q(u)=\operatorname{sign}(u-c)(f(u)-f(c)) .
$$

To this end, we start by observing that the number of fronts is bounded by $\mathcal{O}(1 / h)$ because $F\left(u^{h}\right)$ is uniformly bounded in $h$. In this proof we shall use the notation $z_{l}, z_{r}$ and $u_{l}, u_{r}$ to denote the left and right states of a given front. Furthermore we let $\mathfrak{f}$ denote a front. In this notation

$$
\left|u^{h}\right|_{B V(\mathbf{R})}=\sum_{\mathfrak{f}}\left|u_{r}-u_{l}\right| .
$$

Next let $\mathcal{T}_{\varepsilon}$ denote the fronts that are such that both $\left|z_{l}\right|$ and $\left|z_{r}\right|$ are less than or equal to $\varepsilon$, i.e.,

$$
\mathcal{T}_{\varepsilon}=\left\{\mathfrak{f} \mid \max \left\{\left|z_{l}\right|,\left|z_{r}\right|\right\} \leq \varepsilon\right\} .
$$

Now we have that for fronts $\mathfrak{f}$ such that $\mathfrak{f} \notin \mathcal{T}_{\varepsilon}$,

$$
\left|u_{l}-u_{r}\right| \leq \mathcal{O}\left(\frac{1}{\varepsilon^{2}}\right)\left|z_{l}-z_{r}\right|
$$


Therefore

$$
\begin{aligned}
\left|u^{h}\right|_{B V(\mathbf{R})} & =\sum_{\mathfrak{f} \in \mathcal{T}_{\varepsilon}}\left|u_{l}-u_{r}\right|+\sum_{\mathfrak{f} \notin \mathcal{T}_{\varepsilon}}\left|u_{l}-u_{r}\right| \\
& \leq \sum_{\mathfrak{f} \in \mathcal{T}_{\varepsilon}}\left(\left|u_{l}\right|+\left|u_{r}\right|\right)+\mathcal{O}\left(\frac{1}{\varepsilon^{2}}\right) \sum_{\mathfrak{f} \notin \mathcal{T}_{\varepsilon}}\left|z_{l}-z_{r}\right| \\
& \leq C\left(\frac{\varepsilon^{1 / 2}}{h}+\frac{1}{\varepsilon^{2}}\right),
\end{aligned}
$$

for some constant $C$ that does not depend on $h$. Choosing $\varepsilon=h^{2 / 5}$ gives

$$
\left|u^{h}\right|_{B V(\mathbf{R})} \leq \frac{C}{h} h^{1 / 5}=\frac{C}{h^{4 / 5}} .
$$

Next, let $\left\{x_{i}\right\}$ denote the discontinuities in $k^{h}$, and set $x_{i+1 / 2}=\left(x_{i}+x_{i+1}\right) / 2$. Since $u^{h}$ is an entropy solution in the strip $\Pi_{T}^{i+1 / 2}=\left(x_{i}, x_{i+1}\right) \times(0, T)$ of

$$
u_{t}^{h}+f^{h}\left(u^{h}\right)_{x}=0, \quad u^{h}(x, 0)=u_{0}^{h}(x),
$$

we find that

$$
\begin{aligned}
& \sum_{i} \iint_{\Pi_{T}^{i+1 / 2}} \eta\left(u^{h}\right) \varphi_{t}+q\left(u^{h}\right) \varphi_{x} d x d t+\int_{\mathbf{R}} \eta\left(u_{0}^{h}\right) \varphi(x, 0) d x \\
& \quad+\sum_{i} \int_{0}^{T} \varphi\left(x_{i}, t\right)\left(q_{i}^{+}-q_{i}^{-}\right) d t \geq 0, \quad 0 \leq \varphi \in C_{0}^{\infty}(\mathbf{R} \times[0, T)),
\end{aligned}
$$

where $\eta^{h}=\eta\left(u^{h}\right), q^{h}=q\left(u^{h}\right)$, and $q_{i}^{ \pm}=\lim _{x \rightarrow x_{i} \pm} q^{h}\left(u^{h}(x, t)\right)$. We define $u_{i}^{ \pm}, f_{i}^{ \pm}, \Phi_{i}^{ \pm}$similarly. Since $u^{h} \rightarrow u$ and $q^{h} \rightarrow q$, (3.10) converges to

$$
\int_{0}^{T} \int_{\mathbf{R}} \eta(u) \varphi_{t}+q(u) \varphi_{x} d x d t+\int_{\mathbf{R}} \eta\left(u_{0}\right) \varphi(x, 0) d x
$$

as $h \rightarrow 0$. Next, we consider the second term, (3.11). Note that for a fixed $t$, at each $x_{i}$, there is a either an $a$-front or an aua front. We can regard an aua-front as three separate fronts, first an $a$-front from $\left(z_{l}, a_{l}\right)$ to $\left(z_{m l}, a_{m}\right)$ then a $u$-front to $\left(z_{m r}, a_{m}\right)$ and final an $a$-front to $\left(z_{r}, a_{r}\right)$. In this case $a_{l}>a_{m}>a_{r}$. Therefore we can regard an aua-front as two $a$-fronts with a $u$-front in between, located at the same point. Consequently, in our bookkeeping, we count the location at $a u a$-fronts twice, and insert the (fictitious) value $a_{m}$ in between. The advantage of doing this is that we do not need to consider aua-fronts separately. Note that (3.10) and (3.11) still hold with this relabeling.

If there is an $a$-front at $x_{i}$ we have that

$$
\Phi_{i}^{+}-\Phi_{i}^{-}=a_{i}^{+}-a_{i}^{-}=k^{h}\left(x_{i}+\right)-k^{h}\left(x_{i}-\right) .
$$

Now $f^{\prime}$ does not change sign over the discontinuity, hence by the mean value theorem there is a $\bar{u}_{i}$ between $u_{i}^{+}$and $u_{i}^{-}$such that

$$
\Phi_{i}^{+}-\Phi_{i}^{-}=\int_{u_{i}^{-}}^{u_{i}^{+}} \frac{f^{\prime}(w)}{g(w)} d w=\frac{f_{i}^{+}-f_{i}^{-}}{g\left(\bar{u}_{i}\right)} .
$$

Thus for $a$-fronts,

$$
f_{i}^{+}-f_{i}^{-}=g\left(\bar{u}_{i}\right)\left(a_{i}^{+}-a_{i}^{-}\right) .
$$

Hence if $\operatorname{sign}\left(u_{i}^{+}-c\right)=\operatorname{sign}\left(u_{i}^{-}-c\right)$, then also sign $\left(u_{i}^{+}-c\right)=\operatorname{sign}\left(\bar{u}_{i}-c\right)$ and

$$
q_{i}^{+}-q_{i}^{-}=\operatorname{sign}\left(\bar{u}_{i}-c\right) g\left(\bar{u}_{i}\right)\left(a_{i}^{+}-a_{i}^{-}\right) .
$$


If $\operatorname{sign}\left(u_{i}^{+}-c\right) \neq \operatorname{sign}\left(u_{i}^{-}-c\right)$, we can regard the $a$-front at $x_{i}$ as two separate $a$-fronts both at $x_{i}$. In the middle we insert the fictitious values $z_{m}=c$ and

$$
a_{m}=\frac{1}{z_{i}^{+}-z_{i}^{-}}\left(a_{i}^{-}\left(z_{i}^{+}-z(c)\right)+a_{i}^{+}\left(z(c)-z_{i}^{-}\right)\right) .
$$

Using such fictitious states and counting the associated $x_{i}$ twice, we find that (3.12) holds $\forall i$.

Let $\Delta x_{i}=x_{i+1 / 2}-x_{i-1 / 2}$. We can rewrite the integrand in (3.11) as

$$
\begin{aligned}
\sum_{i}\left(a_{i}^{+}-a_{i}^{-}\right) \operatorname{sign}\left(\bar{u}_{i}-c\right) g\left(\bar{u}_{i}\right) \varphi\left(x_{i}, t\right) \\
\quad=\sum_{i} \int_{x_{i-1 / 2}}^{x_{i+1 / 2}} \frac{a_{i}^{+}-a_{i}^{-}}{\Delta x_{i}} \operatorname{sign}\left(\bar{u}_{i}-c\right) g\left(\bar{u}_{i}\right) \varphi\left(x_{i}, t\right) d x .
\end{aligned}
$$

We now construct $k^{h}$ such that $\max _{i} \Delta x_{i}=\mathcal{O}(h)$ as $h \rightarrow 0$, and

$$
\sum_{i} \frac{a_{i}^{+}-a_{i}^{-}}{\Delta x_{i}} \varphi\left(x_{i}, t\right) \chi_{\left[x_{i-1 / 2}, x_{i+1 / 2}\right)}(x) \rightarrow k^{\prime}(x) \varphi(x, t)
$$

in $L^{1}(\mathbf{R})$ as $h \rightarrow 0$. We use $\chi_{A}$ to denote the characteristic function of a set $A$. It remains to study sign $\left(\bar{u}_{i}-c\right) g\left(\bar{u}_{i}\right) \chi_{\left[x_{i-1 / 2}, x_{i+1 / 2}\right)}(x)$. For a fixed $t$ set

$$
\begin{aligned}
\bar{u}^{h}(x) & =\sum_{i} \bar{u}_{i} \chi_{\left[x_{i-1 / 2}, x_{i+1 / 2}\right)}(x), \\
\Delta \bar{k}^{h}(x) & =\sum_{i} \frac{a_{i}^{+}-a_{i}^{-}}{\Delta x_{i}} \chi_{\left[x_{i-1 / 2}, x_{i+1 / 2}\right)}(x), \\
\bar{\varphi}^{h}(x, t) & =\sum_{i} \varphi\left(x_{i}, t\right) \chi_{\left[x_{i-1 / 2}, x_{i+1 / 2}\right)}(x) .
\end{aligned}
$$

Then

$$
\begin{aligned}
\int_{x_{i-1 / 2}}^{x_{i+1 / 2}}\left|\bar{u}_{i}-u^{h}\right| d x & \leq \int_{x_{i-1 / 2}}^{x_{i+1 / 2}}\left|\bar{u}_{i}-u^{h}\left(x_{i}-, t\right)\right|+\left|u^{h}\left(x_{i}-, t\right)-u^{h}(x, t)\right| d x \\
& \leq \Delta x_{i}\left(\mathcal{O}\left(h^{1 / 2}\right)+\left|u^{h}(\cdot, t)\right|_{B V\left(\left[x_{i-1 / 2}, x_{i+1 / 2}\right)\right)}\right) .
\end{aligned}
$$

Consequently, there is a constant $C$ independent of $h$ such that

$$
\left\|\bar{u}^{h}(\cdot, t)-u^{h}(\cdot, t)\right\|_{L_{\mathrm{loc}}^{1}(\mathbf{R})} \leq C\left(h^{1 / 2}+h^{1 / 5}\right),
$$

and therefore $\bar{u}^{h} \rightarrow u$ in $L_{\text {loc }}^{1}\left(\Pi_{T}\right)$ as $h \rightarrow 0$. Furthermore, with this notation, the sum in (3.11) reads

$$
\int_{0}^{T} \int_{\mathbf{R}} \operatorname{sign}\left(\bar{u}^{h}-c\right) \Delta \bar{k}^{h} g\left(\bar{u}^{h}\right) \bar{\varphi}^{h} d x d t .
$$

Thanks to Lemma 3.2, it therefore follows that the sum in (3.11) converges as $h \rightarrow 0$ to

$$
\int_{0}^{T} \int_{\mathbf{R}} \operatorname{sign}(u-c) g(u) k^{\prime}(x) \varphi d x d t
$$

for any $c \in \mathbf{R} \backslash \Theta$, where $\Theta$ is an at most countable set. Hence, the front tracking limit $u$ satisfies (3.2) for all nonnegative $\varphi \in C_{0}^{\infty}(\mathbf{R} \times[0, T))$ and for all constants $c \in \mathbf{R} \backslash \Theta$. We claim that this actually holds for all $c \in \mathbf{R}$. To this end, we start by writing (3.2) in the form

$$
\int_{0}^{T} \int_{\mathbf{R}} \operatorname{sign}(u-c) g(u) k^{\prime}(x) \varphi d x d t \leq \mathcal{I}(c), \quad c \in \mathbf{R} \backslash \Theta,
$$

where

$$
\mathcal{I}(c):=-\int_{0}^{T} \int_{\mathbf{R}}|u-c| \varphi_{t}+\operatorname{sign}(u-c)(f(u)-f(c)) \varphi_{x} d x d t-\int_{\mathbf{R}}\left|u_{0}-c\right| \varphi(x, 0) d x .
$$


Clearly, $\mathcal{I}(c)$ is a continuous function of $c \in \mathbf{R}$. We want to show that (3.15) also holds for $c \in \Theta$, so fix $c \in \Theta$, and let

$$
\mathcal{E}_{c}=\left\{(x, t) \in \Pi_{T} \mid u(x, t)=c\right\} .
$$

By Lemma 3.2, there are two sequences $\left\{\underline{c}_{\nu}\right\}_{\nu=1}^{\infty},\left\{\bar{c}_{\nu}\right\}_{\nu=1}^{\infty} \subset \mathbf{R} \backslash \Theta$ and

$$
\begin{array}{lll}
\underline{c}_{\nu}<c, & \underline{c}_{\nu} \uparrow c \text { as } \nu \rightarrow \infty, & \operatorname{sign}\left(u-\underline{c}_{\nu}\right) \rightarrow \operatorname{sign}(u-c) \text { a.e. in } \Pi_{T} \backslash \mathcal{E}_{c}, \\
\bar{c}_{\nu}>c, & \bar{c}_{\nu} \downarrow c \text { as } \nu \rightarrow \infty, & \operatorname{sign}\left(u-\bar{c}_{\nu}\right) \rightarrow \operatorname{sign}(u-c) \text { a.e. in } \Pi_{T} \backslash \mathcal{E}_{c} .
\end{array}
$$

Since inequality (3.15) holds for each $\underline{c}_{\nu}$,

$$
\iint_{\Pi_{T} \backslash \mathcal{E}_{c}} \operatorname{sign}\left(u-\underline{c}_{\nu}\right) g(u) k^{\prime}(x) \varphi d x d t+\iint_{\mathcal{E}_{c}} \operatorname{sign}\left(u-\underline{c}_{\nu}\right) g(u) k^{\prime}(x) \varphi d x d t \leq \mathcal{I}\left(\underline{c}_{\nu}\right) .
$$

For $(x, t) \in \mathcal{E}_{c}, \underline{c}_{\nu}<c=u(x, t)$, and therefore

$$
\iint_{\Pi_{T} \backslash \mathcal{E}_{c}} \operatorname{sign}\left(u-\underline{c}_{\nu}\right) g(u) k^{\prime}(x) \varphi d x d t+\iint_{\mathcal{E}_{c}} g(u) k^{\prime}(x) \varphi d x d t \leq \mathcal{I}\left(\underline{c}_{\nu}\right) .
$$

Using this and (3.16) when sending $\underline{c}_{\nu} \uparrow c$ in (3.17) yields

$$
\iint_{\Pi_{T} \backslash \mathcal{E}_{c}} \operatorname{sign}(u-c) g(u) k^{\prime}(x) \varphi d x d t+\iint_{\mathcal{E}_{c}} g(u) k^{\prime}(x) \varphi d x d t \leq \mathcal{I}(c) .
$$

A similar calculation using $\bar{c}_{\nu}$ yields the inequality

$$
\iint_{\Pi_{T} \backslash \mathcal{E}_{c}} \operatorname{sign}(u-c) g(u) k^{\prime}(x) \varphi d x d t-\iint_{\mathcal{E}_{c}} g(u) k^{\prime}(x) \varphi d x d t \leq \mathcal{I}(c) .
$$

By adding (3.18) and (3.19) and then dividing by two, we get

$$
\iint_{\Pi_{T} \backslash \mathcal{E}_{c}} \operatorname{sign}(u-c) g(u) k^{\prime}(x) \varphi d x d t \leq \mathcal{I}(c)
$$

Since $\operatorname{sign}(0)=0$,

$$
\operatorname{sign}(u-c) g(u) k^{\prime}(x) \varphi=0 \quad \text { on } \mathcal{E}_{c},
$$

so that we can extend the domain of integration to all of $\Pi_{T}$ :

$$
\int_{0}^{T} \int_{\mathbf{R}} \operatorname{sign}(u-c) g(u) k^{\prime}(x) \varphi d x d t \leq \mathcal{I}(c) .
$$

We have shown that entropy inequality (3.2) holds for $c \in \Theta$, and thus by (3.15) for all $c \in \mathbf{R}$. This concludes the proof of Theorem 3.2.

Corollary 3.1 (convergence result). Suppose $f, g, k, u_{0}$ satisfy the conditions stated in Section 1. Let $u^{h}=u^{h}(x, t)$ be the front tracking solution defined in Section 2. Let $u=u(x, t)$ be the unique entropy solution of (1.1). Then $u^{h} \rightarrow u$ in $L_{\mathrm{loc}}^{1}\left(\Pi_{T}\right)$ as $h \rightarrow 0$.

Proof. This is a consequence of Theorem 3.1, Theorem 3.2, and Kružkov uniqueness result [48].

\section{Numerical EXAMPLES}

In this section we present three numerical examples that demonstrate the properties of our front tracking method. In the numerical examples we use, as before,

$$
\text { the flux function } f(u)=4 u(1-u) \text { and the source term } g(u)=u \text {. }
$$

To compare the front tracking computations with a reference solution we use the standard EngquistOsher scheme [13] defined by

$$
u_{j}^{n+1}=u_{j}^{n}-\lambda\left(f^{\mathrm{EO}}\left(u_{j+1}^{n}, u_{j}^{n}\right)-f^{\mathrm{EO}}\left(u_{j}^{n}, u_{j-1}^{n}\right)\right)+u_{j}^{n} k^{\prime}\left(x_{j}\right) \Delta t,
$$

where the Engquist-Osher flux $f^{\mathrm{EO}}$ reads

$$
f^{\mathrm{EO}}(u, v)=f(\max \{1 / 2, u\})+f(\min \{1 / 2, v\}),
$$




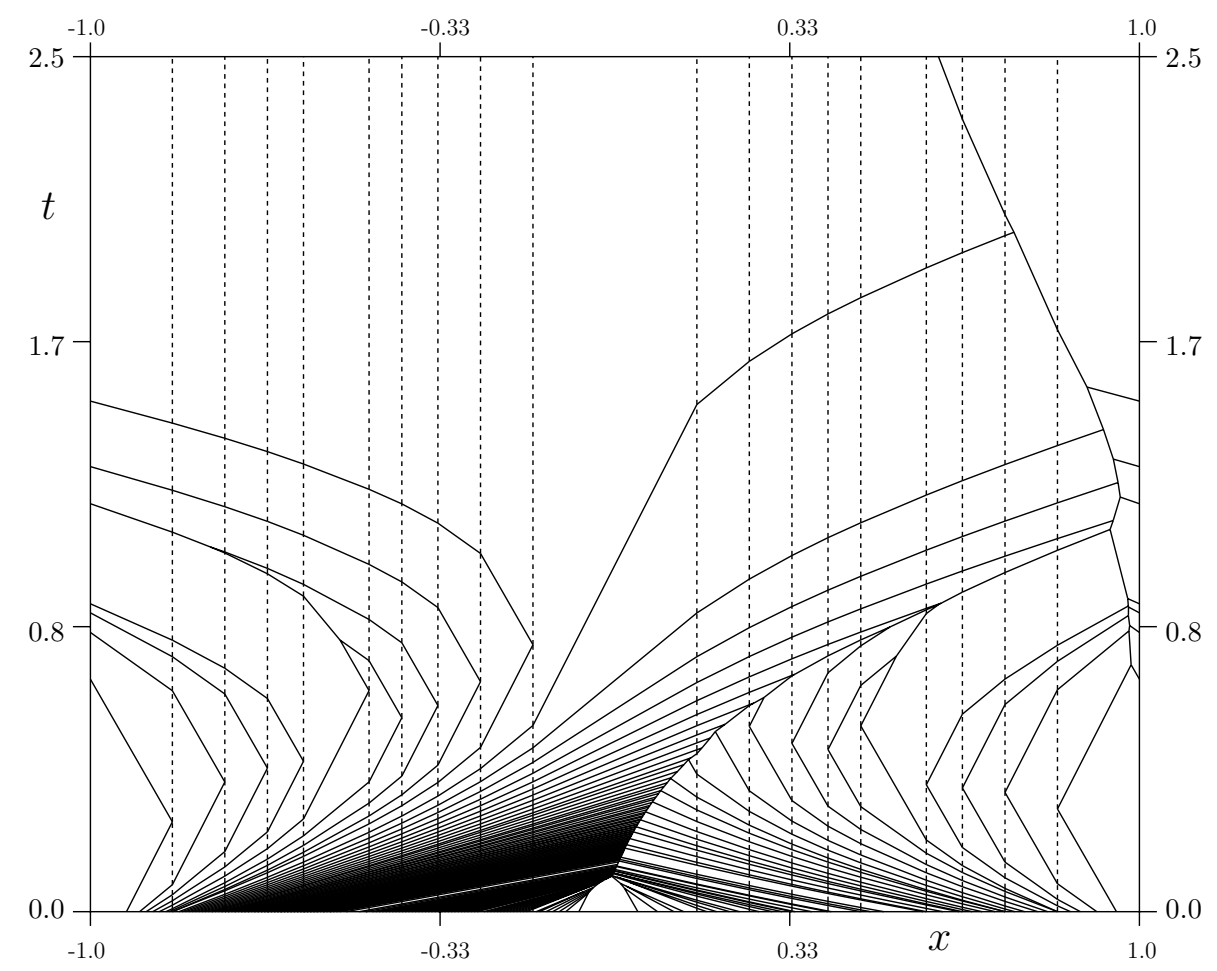

Figure 3. (Example 1) Fronts in the front tracking solution $u^{h}(\cdot, t)$ for times $t \in[0,2.5]$. Solid lines: $u$-fronts. Broken lines: $a$-fronts.

where $f$ is given by (4.1). Here $x_{j}=j \Delta x$, and $\lambda=\Delta t / \Delta x$, and we assume that the CFL-condition

$$
\lambda<\max _{j}\left|f^{\prime}\left(u_{j}^{n}\right)\right|
$$

is satisfied.

We also compare the front tracking approximations with approximations generated by operator splitting. This splitting uses front tracking for the hyperbolic part of the problem, and an ODE solver for the ordinary differential equation $u_{t}=g(u)$.

Example 1 (non-stiff source). In this example we use

$$
u_{0}(x)=0.5(1+0.9 \sin (\pi x)), \quad k(x)=\frac{c}{\pi} \cos (\pi x),
$$

where $c=1$. This problem is not stiff, and one would expect methods based on operator splitting to be satisfactory for this problem. In Figure 3 we show the fronts in the front tracking approximation, for $h=1 / 16$. Figure 4 shows the initial approximation, i.e., $u^{h}(x, 0)$ and $u^{\delta}(x, 2.5)$. Table 1 shows the relative errors defined by

$$
\text { Error }=100 \times \frac{\| \text { approximate }- \text { reference } \|_{L^{1}}}{\| \text { reference } \|_{L^{1}}} .
$$

for the front tracking method, as well as for the operator splitting method based on front tracking. The table also shows the CPU-time ${ }^{1}$ in seconds. We compared the solutions at $t=5.0$. The splitting method used a spatial grid with grid spacing $\Delta x=\delta / 10$. As a reference solution we used the Engquist-Osher scheme with 8196 grid points in the interval $[-1,1]$. The data in Table 1 indicate that a numerical convergence rate for the tracking method of about 1 , and for the splitting method of $1.7(\mathrm{CFL}=10)$ and $2.7(\mathrm{CFL}=50)$. In Figure 5 we show the relationship between the

\footnotetext{
${ }^{1}$ All computations were done on a PowerBook G3, 500MHz, and the CPU times reported include only the computations, not the time used for initializations and memory allocations. All algorithms were coded in the "C" programming language and compiled with Metrowerks CodeWarrior 6.0 with optimization level 2.
} 

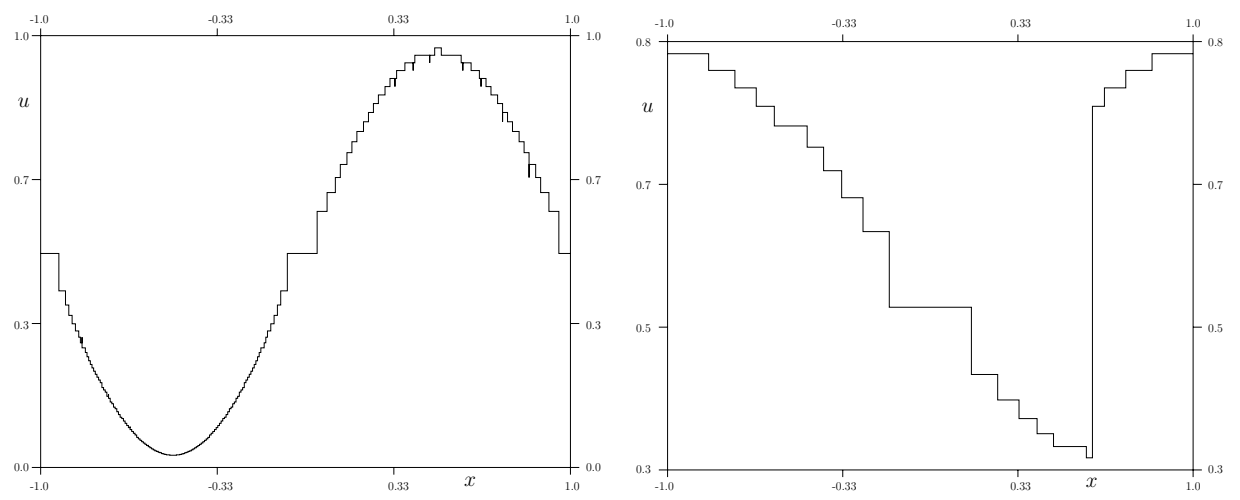

Figure 4. (Example 1) Left: $u^{h}(\cdot, 0)$. Right: $u^{h}(\cdot, 2.5)$.

TABLE 1. Errors and CPU-times for Example 1.

\begin{tabular}{|c|r|r|r|r|r|r|}
\hline & \multicolumn{2}{|c|}{ Tracking } & \multicolumn{4}{c|}{ Splitting } \\
\hline & \multicolumn{2}{|c|}{} & \multicolumn{2}{|c|}{ CFL $=10.0$} & \multicolumn{2}{c|}{ CFL $=50$} \\
\hline $1 / h$ & Error & CPU & Error & CPU & Error & CPU \\
\hline 2 & 9.75 & 0.01 & 14.48 & 0.01 & 681.22 & 0.01 \\
\hline 4 & 14.44 & 0.01 & 2.66 & 0.02 & 177.44 & 0.01 \\
\hline 8 & 4.24 & 0.03 & 0.65 & 0.07 & 18.49 & 0.03 \\
\hline 16 & 3.06 & 0.08 & 0.17 & 0.28 & 3.87 & 0.18 \\
\hline 32 & 1.85 & 0.47 & 0.05 & 1.82 & 0.63 & 1.45 \\
\hline 64 & 0.34 & 27.88 & 0.05 & 6.97 & 0.06 & 25.18 \\
\hline
\end{tabular}

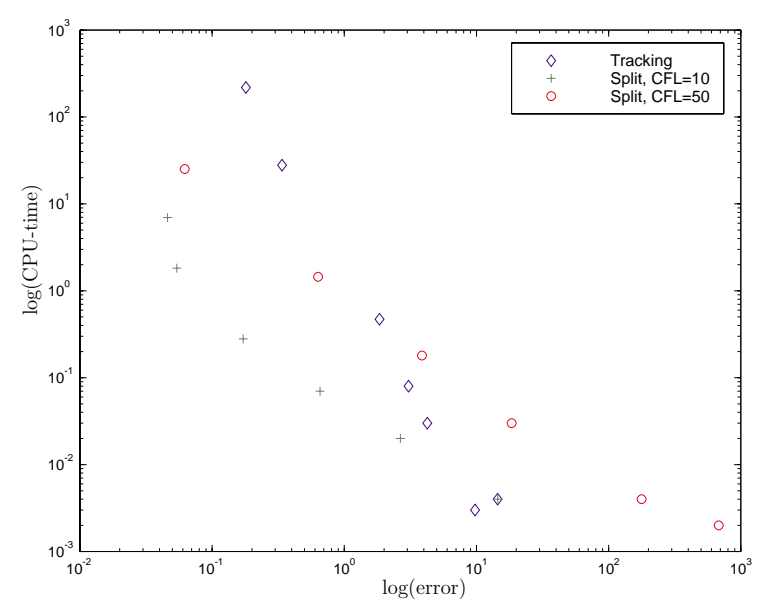

FiguRE 5. (Example 1) Logarithmic plot of the error versus the CPU-time.

errors and the CPU-time for the three methods. From Table 1 and Figure 5 we conclude that the splitting method performs (slightly) better than the front tracking method for this example.

Example 2 (stiff source). Changing the constant $c$ in (4.2) to 50 makes the problem "semistiff". This poses no conceptual problem for the front tracking method, but methods based on operator splitting will have a harder time approximating the solution, although they still converge. In Figure 6 we show the approximate solutions made by front tracking, operator splitting with $\mathrm{CFL}=1$ and $\mathrm{CFL}=10$, as well as the reference solution produced by a semi-implicit Engquist-Osher 


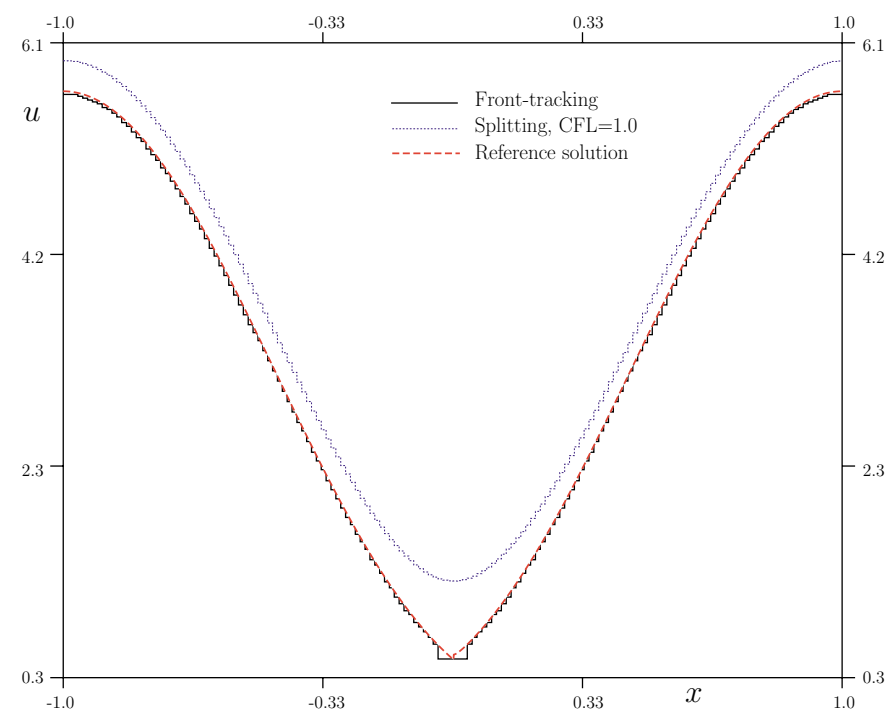

FiguRE 6. (Example 2) Front tracking and operator splitting.

TABle 2. Errors and CPU-times for Example 2.

\begin{tabular}{|c|r|r|r|r|r|r|}
\hline & \multicolumn{2}{|c|}{ Tracking } & \multicolumn{4}{|c|}{ Splitting } \\
\hline & \multicolumn{2}{|c|}{} & \multicolumn{2}{|c|}{ CFL=1 } & \multicolumn{2}{c|}{ CFL $=10$} \\
\hline $1 / h$ & Error & CPU & Error & CPU & Error & CPU \\
\hline 2 & 2.4 & 0.02 & 43.9 & 0.08 & 1050.4 & 0.10 \\
\hline 4 & 1.2 & 0.08 & 22.1 & 0.33 & 11.1 & 0.05 \\
\hline 8 & 0.8 & 0.50 & 10.2 & 1.32 & 5.7 & 0.10 \\
\hline 16 & 0.3 & 3.78 & 4.6 & 5.32 & 2.8 & 0.77 \\
\hline 32 & 0.2 & 28.62 & 1.8 & 25.23 & 1.2 & 4.79 \\
\hline 64 & 0.1 & 211.93 & 0.6 & 101.85 & 0.7 & 17.37 \\
\hline
\end{tabular}

method. ${ }^{2}$ We compared the methods at $t=1$, and the front tracking and the operator splitting approximations used $h=1 / 16$. Since this problem is "stiffer" than the previous problem, the method based on operator splitting was not able to handle CFL-numbers larger than about 20. Table 2 shows the errors and the CPU-time for this example. This table indicates that the front tracking method has a numerical convergence rate of 0.9 with respect to $h$, while the splitting method exhibited numerical convergence rates of $1.2(\mathrm{CFL}=1)$ and $1.8(\mathrm{CFL}=10)$. In Figure 7 we show the relationship between the errors and the CPU-time for the three methods. In contrast to Figure 5 we see that the front tracking method consistently uses less CPU-time to produce a given error. Hence, for this example we must conclude that the front tracking method performs better than the splitting method.

Example 3 (steady-state solution). In our final example we shall demonstrate that the front tracking method can preserve steady-state solutions (with good accuracy), while the simple Engquist-Osher and splitting schemes do not have this property. We use initial data given by

$$
u_{0}(x)=0.75+0.24 \sin (4 \pi x), \quad \text { and } \quad k(x)=4 \ln \left(u_{0}(x) e^{-2 u_{0}(x)}\right) .
$$

Due to the special form of $k$, the exact solution is given by $u(x, t)=u_{0}(x)$. In Figure 8 we see how the front tracking method, the Engquist-Osher scheme, and the splitting scheme with $\mathrm{CFL}=10$ perform on this problem. We show the numerical approximations at $t=6$, and have used $\delta=h=1 / 32$. For this value of $h$, the Engquist-Osher and the split schemes give results

\footnotetext{
${ }^{2}$ We discretized the source term at time $t=t^{n+1}$, which works well since the source term is linear.
} 


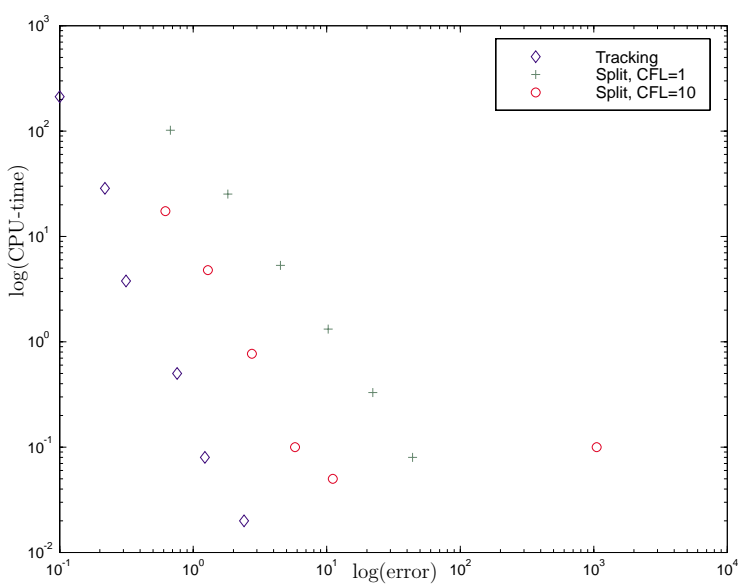

Figure 7. (Example 2) Logarithmic plot of the error versus the CPU-time.

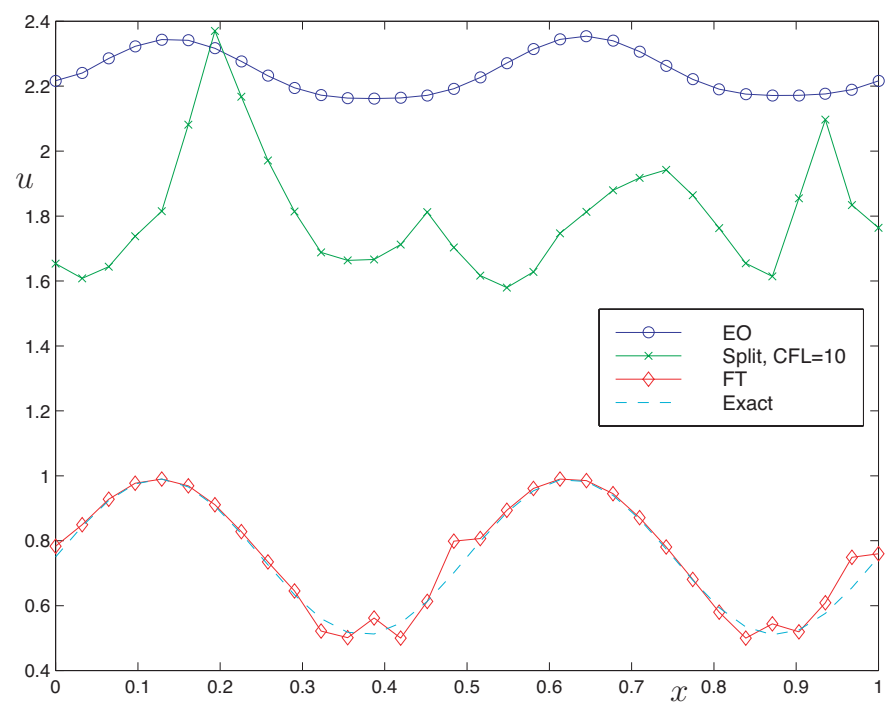

Figure 8. (Example 3) The Engquist-Osher, the splitting, and the front tracking schemes at $t=6$ using $h=1 / 32$.

that are clearly too inaccurate to be meaningful. In Table 3 we show the relative $L^{1}$ errors for various values of $h$. For the front tracking scheme we have used $\delta=h$. Although the front tracking scheme uses the Riemann solution, it is not exactly stationary for this problem, since this scheme involves projecting the initial data onto a grid in the $(u, k)$ plane. This projection does not leave "stationary" initial data constant, and the initial error for the front tracking method is usually larger than the final error. Table 3 shows that both the splitting and the Engquist-Osher cannot calculate asymptotic stationary values, unless $h$ is very small.

\section{Conclusion}

We have presented a front tracking method for numerically solving scalar conservation laws with source term. The method is not based on operator splitting to "split off" the effect of the source term, as is usually done in the literature. Instead we process the source term by building it directly into to the Riemann solver used by front tracking. Using the singular mapping technique, we have proved that the front tracking method converge to the unique Kružkov entropy solution. 
TABLE 3. Errors for Example 3.

\begin{tabular}{|r|r|r|r|r|}
\hline & \multicolumn{2}{|c|}{ Splitting } & & \\
\hline $1 / h$ & $\mathrm{CFL}=5$ & $\mathrm{CFL}=15$ & $\mathrm{EO}$ & $\mathrm{FT}$ \\
\hline 4 & 950.8 & 3284.7 & 1966.4 & 24.7 \\
\hline 5 & 92.3 & 1051.1 & 1008.5 & 13.5 \\
\hline 6 & 40.2 & 227.6 & 476.6 & 2.3 \\
\hline 7 & 19.9 & 43.7 & 231.7 & 0.8 \\
\hline 8 & 10.0 & 21.4 & 114.4 & 1.6 \\
\hline 9 & 5.0 & 10.8 & 56.4 & 0.7 \\
\hline 10 & 2.6 & 5.2 & 27.6 & 0.7 \\
\hline
\end{tabular}

We have demonstrated through numerical examples that the proposed front tracking method can treat stiff source terms as well as preserving steady-state solutions (or achieving them in the long time limit) with good accuracy. The approach proposed herein can be combined with $[33,53]$ to yield a numerical method for multi-dimensional scalar conservation laws with source term. To deal with systems like those in $[32,29,27]$ we need to build appropriate Riemann solvers that take into account the effect of the source term. This is left as interesting problems for future research.

\section{REFERENCES}

[1] F. Alcrudo and F. Benkhaldoun. Exact solutions to the Riemann problem of the shallow water equations with a bottom step. Comput. \& Fluids, 30(6):643-671, 2001.

[2] D. Amadori, L. Gosse, and G. Guerra. Global BV entropy solutions and uniqueness for hyperbolic systems of balance laws. Arch. Ration. Mech. Anal., 162(4):327-366, 2002.

[3] A. Bermúdez, A. Dervieux, J.-A. Desideri, and M. E. Vázquez. Upwind schemes for the two-dimensional shallow water equations with variable depth using unstructured meshes. Comput. Methods Appl. Mech. Engrg., 155(12):49-72, 1998.

[4] A. Bermudez and M. E. Vazquez. Upwind methods for hyperbolic conservation laws with source terms. Comput. Ef Fluids, 23(8):1049-1071, 1994.

[5] R. Botchorishvili, B. Perthame, and A. Vasseur. Equilibrium schemes for scalar conservation laws with stiff sources. Math. Comp., 72(241):131-157 (electronic), 2003.

[6] F. Bratvedt, K. Bratvedt, C. F. Buchholz, T. Gimse, H. Holden, L. Holden, and N. H. Risebro. FRONTLINE and FRONTSIM: two full scale, two-phase, black oil reservoir simulators based on front tracking. Surveys Math. Indust., 3(3):185-215, 1993.

[7] A. Bressan. Global solutions of systems of conservation laws by wave-front tracking. J. Math. Anal. Appl., 170(2):414-432, 1992.

[8] A. Bressan. Hyperbolic systems of conservation laws, volume 20 of Oxford Lecture Series in Mathematics and its Applications. Oxford University Press, Oxford, 2000. The one-dimensional Cauchy problem.

[9] M. Castro, J. Macías, and C. Parés. A $Q$-scheme for a class of systems of coupled conservation laws with source term. Application to a two-layer 1-D shallow water system. M2AN Math. Model. Numer. Anal., 35(1):107-127, 2001.

[10] G.-Q. Chen and J. Glimm. Global solutions to the compressible Euler equations with geometrical structure. Comm. Math. Phys., 180(1):153-193, 1996.

[11] C. M. Dafermos. Polygonal approximations of solutions of the initial value problem for a conservation law. $J$. Math. Anal. Appl., 38:33-41, 1972.

[12] R. J. DiPerna. Global existence of solutions to nonlinear hyperbolic systems of conservation laws. J. Differential Equations, 20(1):187-212, 1976.

[13] B. Engquist and S. Osher. One-sided difference approximations for nonlinear conservation laws. Math. Comp., 36(154):321-351, 1981.

[14] M. S. Espedal and K. H. Karlsen. Numerical solution of reservoir flow models based on large time step operator splitting algorithms. In Filtration in Porous Media and Industrial Applications (Cetraro, Italy, 1998), volume 1734 of Lecture Notes in Mathematics, pages 9-77. Springer, Berlin, 2000.

[15] T. Gallouët, J. M. Hérard, and N. Seguin. Some approximate godunov schemes to compute shallow-water equations with topography. Computers and Fluids. To appear.

[16] L. Gascón and J. M. Corberán. Construction of second-order TVD schemes for nonhomogeneous hyperbolic conservation laws. J. Comput. Phys., 172(1):261-297, 2001.

[17] T. Gimse and N. H. Risebro. Riemann problems with a discontinuous flux function. In Proc. 3rd Internat. Conf. Hyperbolic Problems, pages 488-502, Uppsala, 1991. Studentlitteratur.

[18] T. Gimse and N. H. Risebro. Solution of the Cauchy problem for a conservation law with a discontinuous flux function. SIAM J. Math. Anal., 23(3):635-648, 1992. 
[19] H. M. Glaz and T.-P. Liu. The asymptotic analysis of wave interactions and numerical calculations of transonic nozzle flow. Adv. in Appl. Math., 5(2):111-146, 1984.

[20] J. Glimm, G. Marshall, and B. Plohr. A generalized Riemann problem for quasi-one-dimensional gas flows. Adv. in Appl. Math., 5(1):1-30, 1984.

[21] L. Gosse. A priori error estimate for a well-balanced scheme designed for inhomogeneous scalar conservation laws. C. R. Acad. Sci. Paris Sér. I Math., 327(5):467-472, 1998.

[22] L. Gosse. A well-balanced flux-vector splitting scheme designed for hyperbolic systems of conservation laws with source terms. Comput. Math. Appl., 39(9-10):135-159, 2000.

[23] L. Gosse. A well-balanced scheme using non-conservative products designed for hyperbolic systems of conservation laws with source terms. Math. Models Methods Appl. Sci., 11(2):339-365, 2001.

[24] L. Gosse and A.-Y. Leroux. Un schéma-équilibre adapté aux lois de conservation scalaires non-homogènes. $C$. R. Acad. Sci. Paris Sér. I Math., 323(5):543-546, 1996.

[25] J. M. Greenberg and A. Y. Leroux. A well-balanced scheme for the numerical processing of source terms in hyperbolic equations. SIAM J. Numer. Anal., 33(1):1-16, 1996.

[26] J. M. Greenberg, A. Y. Leroux, R. Baraille, and A. Noussair. Analysis and approximation of conservation laws with source terms. SIAM J. Numer. Anal., 34(5):1980-2007, 1997.

[27] V. Haugse, K. H. Karlsen, K.-A. Lie, and J. Natvig. Numerical solution of the polymer system by front tracking. Transport in Porous Media. To appear.

[28] C. Helzel, R. J. LeVeque, and G. Warnecke. A modified fractional step method for the accurate approximation of detonation waves. SIAM J. Sci. Comput., 22(4):1489-1510 (electronic), 2000.

[29] R. Holdahl, H. Holden, and K.-A. Lie. Unconditionally stable splitting methods for the shallow water equations. BIT, 39(3):451-472, 1999.

[30] H. Holden and L. Holden. On scalar conservation laws in one-dimension. In S. Albeverio, J. E. Fenstad, H. Holden, and T. Lindstrøm, editors, Ideas and Methods in Mathematics and Physics, pages 480-509. Cambridge University Press, Cambridge, 1988.

[31] H. Holden, L. Holden, and R. Høegh-Krohn. A numerical method for first order nonlinear scalar conservation laws in one dimension. Comput. Math. Appl., 15(6-8):595-602, 1988.

[32] H. Holden, K.-A. Lie, and N. H. Risebro. An unconditionally stable method for the Euler equations. J. Comput. Phys., 150(1):76-96, 1999.

[33] H. Holden and N. H. Risebro. A method of fractional steps for scalar conservation laws without the CFL condition. Math. Comp., 60(201):221-232, 1993.

[34] H. Holden and N. H. Risebro. Conservation laws with a random source. Appl. Math. Optim., 36(2):229-241, 1997.

[35] H. Holden and N. H. Risebro. Front tracking for hyperbolic conservation laws, volume 152 of Applied Mathematical Sciences. Springer-Verlag, New York, 2002.

[36] J. M.-K. Hong. Part I: An extension of the Riemann problems and Glimm's method to general systems of conservation laws with source terms. Part II: A total variation bound on the conserved quantities for a generic resonant nonlinear balance laws. PhD thesis, University of California, Davis, 2000.

[37] M. E. Hubbard and P. Garcia-Navarro. Flux difference splitting and the balancing of source terms and flux gradients. J. Comput. Phys., 165(1):89-125, 2000.

[38] E. Isaacson and B. Temple. Nonlinear resonance in inhomogeneous systems of conservation laws. In Mathematics of nonlinear science (Phoenix, AZ, 1989), volume 108 of Contemp. Math., pages 63-77. Amer. Math. Soc., Providence, RI, 1990.

[39] E. Isaacson and B. Temple. Convergence of the $2 \times 2$ Godunov method for a general resonant nonlinear balance law. SIAM J. Appl. Math., 55(3):625-640, 1995.

[40] E. R. Jakobsen, K. H. Karlsen, and N. H. Risebro. On the convergence rate of operator splitting for HamiltonJacobi equations with source terms. SIAM J. Numer. Anal., 39(2):499-518 (electronic), 2001.

[41] P. Jenny and B. Müller. Rankine-Hugoniot-Riemann solver considering source terms and multidimensional effects. J. Comput. Phys., 145(2):575-610, 1998.

[42] S. Jin. A steady-state capturing method for hyperbolic systems with geometrical source terms. M2AN Math. Model. Numer. Anal., 35(4):631-645, 2001.

[43] K. H. Karlsen. On the accuracy of a dimensional splitting method for scalar conservation laws. Master's thesis, Department of Mathematics, University of Oslo, Norway, 1994.

[44] K. H. Karlsen and K.-A. Lie. An unconditionally stable splitting scheme for a class of nonlinear parabolic equations. IMA J. Numer. Anal., 19(4):609-635, 1999.

[45] K. H. Karlsen and N. H. Risebro. A note on front tracking and the equivalence between viscosity solutions of hamilton-jacobi equations and entropy solutions of scalar conservation laws. Nonlinear Analysis: Theory, Methods \& Applications, 50(4):455-469, 2002.

[46] K. H. Karlsen and N. H. Risebro. Unconditionally stable methods for Hamilton-Jacobi equations. J. Comput. Phys., 180(2):710-735, 2002.

[47] C. Klingenberg and N. H. Risebro. Convex conservation laws with discontinuous coefficients. Existence, uniqueness and asymptotic behavior. Comm. Partial Differential Equations, 20(11-12):1959-1990, 1995.

[48] S. N. Kružkov. First order quasi-linear equations in several independent variables. Math. USSR Sbornik, 10(2):217-243, 1970. 
[49] A. Kurganov and D. Levy. Central-upwind schemes for the Saint-Venant system. M2AN Math. Model. Numer. Anal., 36(3):397-425, 2002.

[50] J. O. Langseth, A. Tveito, and R. Winther. On the convergence of operator splitting applied to conservation laws with source terms. SIAM J. Numer. Anal., 33(3):843-863, 1996.

[51] R. J. LeVeque. Balancing source terms and flux gradients in high-resolution Godunov methods: the quasisteady wave-propagation algorithm. J. Comput. Phys., 146(1):346-365, 1998.

[52] R. J. LeVeque and H. C. Yee. A study of numerical methods for hyperbolic conservation laws with stiff source terms. J. Comput. Phys., 86(1):187-210, 1990.

[53] K.-A. Lie, V. Haugse, and K. Hvistendahl Karlsen. Dimensional splitting with front tracking and adaptive grid refinement. Numer. Methods Partial Differential Equations, 14(5):627-648, 1998.

[54] T. P. Liu. Quasilinear hyperbolic systems. Comm. Math. Phys., 68(2):141-172, 1979.

[55] T. P. Liu. Resonance for quasilinear hyperbolic equation. Bull. Amer. Math. Soc. (N.S.), 6(3):463-465, 1982.

[56] T.-P. Liu. Nonlinear resonance for quasilinear hyperbolic equation. J. Math. Phys., 28(11):2593-2602, 1987.

[57] A. Noussair. Riemann problem with nonlinear resonance effects and well-balanced Godunov scheme for shallow fluid flow past an obstacle. SIAM J. Numer. Anal., 39(1):52-72 (electronic), 2001.

[58] A. Noussair. Riemann problem with nonlinear resonance effects and well-balanced Godunov scheme for shallow fluid flow past an obstacle. SIAM J. Numer. Anal., 39(1):52-72 (electronic), 2001.

[59] M. V. Papalexandris, A. Leonard, and P. E. Dimotakis. Unsplit schemes for hyperbolic conservation laws with source terms in one space dimension. J. Comput. Phys., 134(1):31-61, 1997.

[60] M. V. Papalexandris, A. Leonard, and P. E. Dimotakis. Unsplit algorithms for multidimensional systems of hyperbolic conservation laws with source terms. Comput. Math. Appl., 44(1-2):25-49, 2002.

[61] B. Perthame and C. Simeoni. A kinetic scheme for the Saint-Venant system with a source term. Calcolo, 38(4):201-231, 2001.

[62] N. H. Risebro. A front-tracking alternative to the random choice method. Proc. Amer. Math. Soc., 117(4):11251139, 1993.

[63] N. H. Risebro and A. Tveito. Front tracking applied to a nonstrictly hyperbolic system of conservation laws. SIAM J. Sci. Stat. Comput., 12(6):1401-1419, 1991.

[64] N. H. Risebro and A. Tveito. A front tracking method for conservation laws in one dimension. J. Comp. Phys., 101(1):130-139, 1992.

[65] P. L. Roe. Upwind differencing schemes for hyperbolic conservation laws with source terms. In Nonlinear hyperbolic problems (St. Etienne, 1986), volume 1270 of Lecture Notes in Math., pages 41-51. Springer, Berlin, 1987.

[66] P. K. Sweby. TVD schemes for inhomogeneous conservation laws. In Nonlinear hyperbolic equations-theory, computation methods, and applications (Aachen, 1988), volume 24 of Notes Numer. Fluid Mech., pages 128 138. Vieweg, Braunschweig, 1989.

[67] H. Z. Tang, T. Tang, and K. Xu. A gas-kinetic scheme for shallow-water equations with source terms. Z. Angew. Math. Phys. To appear.

[68] T. Tang. Convergence analysis for operator-splitting methods applied to conservation laws with stiff source terms. SIAM J. Numer. Anal., 35(5):1939-1968 (electronic), 1998.

[69] B. Temple. Global solution of the Cauchy problem for a class of $2 \times 2$ nonstrictly hyperbolic conservation laws. Adv. in Appl. Math., 3(3):335-375, 1982.

[70] B. van Leer. On the relation between the upwind-differencing schemes of Godunov, Engquist-Osher and Roe. SIAM J. Sci. Statist. Comput., 5(1):1-20, 1984.

[71] M. E. Vázquez-Cendón. Improved treatment of source terms in upwind schemes for the shallow water equations in channels with irregular geometry. J. Comput. Phys., 148(2):497-526, 1999.

[72] K. Xu. A well-balanced gas-kinetic scheme for the shallow-water equations with source terms. J. Comput. Phys., 178(2):533-562, 2002.

[73] J. G. Zhou, D. M. Causon, C. G. Mingham, and D. M. Ingram. The surface gradient method for the treatment of source terms in the shallow-water equations. J. Comput. Phys., 168(1):1-25, 2001. 
(Karlsen)

Department of Mathematics

UNIVERSITY OF BERGEN

Johs. BRUngt. 12

N-5008 Bergen, Norway

E-mail address: kennethk@math.uib.no

$U R L$ : www.mi.uib.no/ ₹kennethk

(Risebro)

Department of Mathematics

University of OsLO

P.O. Box 1053, BLINDERN

N-0316 Oslo, Norway

E-mail address: nilshr@math.uio.no

$U R L$ : www.math.uio.no/ nilshr

(Towers)

Mira Costa College

3333 Manchester Avenue

CARdiff-BY-The-SeA, CA 92007-1516, USA

E-mail address: jtowers@cts.com

$U R L$ : www.miracosta.cc.ca.us/home/jtowers/ 